Linköping Studies in Science and Technology

Dissertation No. 2174

\title{
Expanding the versatility \\ and functionality of \\ iontronic devices
}

Dennis Cherian 

Doctor of Philosophy

Doctoral thesis in Applied Physics

Norrköping, 2021

\section{Expanding the versatility and functionality of iontronic devices}

Dennis Cherian

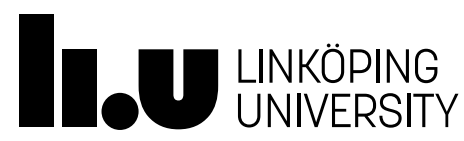


Description of the cover page: Depicts an ion separation device described in the thesis. Photo taken by Johannes Gladisch and edited by Dennis Cherian, LiU.

Copyright $\odot 2021$ Dennis Cherian

(c) (i)

This work is licensed under a Creative Commons Attribution Non-Commercial 4.0 International License (www.creativecommons.org/licenses/by/4.0/).

ISBN: 978-91-7929-035-1

ISSN: 0345-7524

Printed in Sweden by LiU-Tryck, Linköping, 2021

Electronic publication:www.ep.liu.se

Laboratory of Organic Electronics Department of Science and Technology Linköping University

Campus Norrköping 60174 Norrköping, Sweden 


\section{Abstract}

Biological systems rarely use electrons as signal regulators, most of the transport and communication in these system utilize ions. The discovery of conjugated polymers and polyelectrolytes and their unique properties of mixed ionic electronic properties opened the possibility of using these in the domain of bioelectronics, which paved the way for the field of organic bioelectronics. After the introduction of the organic electronic ion pump (OEIP) in 2007, which utilizes both the ionic properties of conjugated polymers and polyelectrolytes, the new field of "iontronics" evolved. The OEIP is an organic polymer-based delivery system based on electrophoretic transport of biologically relevant and ionically charged species, without fluid flow and with high spatial, temporal, and dosage precision. These devices have been extensively studied for the past 14 years and have found numerous demonstrations in in vivo and in vitro delivery of bio-relevant ions for therapeutic application. This has, in parallel, resulted in the development of custom materials for ion exchange membranes (IEMs) within the OEIP.

This thesis focuses on IEMs and device development of OEIPs. Specific focus is given to process development through device design and fabrication through conventional and unconventional technologies. Conventional technologies include microfabrication through photolithography, etching, and thin-film evaporation. Unconventional fabrication techniques include screen printing, inkjet printing, stencil, and laser patterning. In this thesis, we have also scouted a new area of research to utilize the ion-selective properties of polyelectrolytes. Here we discuss a new ion detection technique using IEMs and ion transport based on diffusion coefficients and impedance measurement at a specific frequency using impedance spectroscopy for faster ion detection with low voltage $(1-40 \mathrm{~V})$ and liquid-flow-free transport. Further exploring the area of IEMs, we have realized that less attention has been given to stretchable IEMs, even though such materials could find enormous applications in the field of organic bioelectronics and can be used in association with many stretchable electronics applications like stretchable displays and energy storage devices. Current IEMs lack the conformability and stretchability to be used for implantable applications, e.g., including lungs, heart, muscle, soft or brain implants, joints, etc. Keeping this in mind we also discuss our approach for the development of a stretchable IEM. Finally, we focus on developing a hybrid fabrication protocol of flexible OEIPs with micropatterning techniques and inkjet-printed membranes. These OEIPs were fabricated and the functionality was validated by the cell response after the delivery of a nerve-blocking 
agent to cells in vitro. To date, OEIPs have been fabricated by micropatterning and labor-intensive manual techniques, impeding the budding application areas of this propitious technology. To address this issue, a novel approach to the fabrication of the OEIPs using screen-printing technology is also explored in this thesis.

In summary, we were able to successfully explore the field of ion exchange membranes and put forward a new technique for ion detection and stretchable IEMs for future applications. Fabrication of OEIPs was also examined which resulted in the development of a hybrid fabrication protocol with inkjet printing for OEIPs and a robust fully screen printed OEIPs with high manufacturing yield (>90\%) for industrial-scale manufacturing. 


\section{Populärvetenskaplig Sammanfattning}

Det biologiska systemet använder sällan elektroner som signaljusterare, den mesta av transporten och kommunikationen i detta system sker igenom joner. Upptäckten av konjugerade polymerer och polyelektrolyter och deras unika egenskaper för blandade joniska och elektroniska egenskaper öppnade möjligheten att använda dessa inom bioelektronik, vilket gräver en väg för området organisk bioelektronik. Efter introduktionen av den organiska elektroniska jonpumpen (OEIP) 2007, som använder egenskapen hos dessa konjugerade polymerer och polyelektrolyter med jonisk selektivitet, utvecklades ett nytt fält som kallas Iontronics. OEIP är ett organiskt polymerbaserat leveranssystem baserat på elektroforetisk transport av biologiskt relevanta anjoniska eller katjoniskt laddade arter utan vätskeflöde och med hög rumslig, tidsmässig och doseringsprecision. Dessa enheter har studerats omfattande under de senaste 14 åren och det har funnits enorm applikation i in-vivo och in-vitro leverans av biorelevanta joner för terapeutisk applikation. Detta resulterar parallellt i utvecklingen av material för jonbytarmembran (IEM).

Denna avhandling fokuserar på jonbytarmembran (IEM) och enhetsutveckling av OEIP. I denna avhandling undersöker vi ett nytt forskningsområde för att utnyttja de jonselektiva egenskaperna hos polyelektrolyter. Här diskuterar vi en ny vätskefri jondetekteringsteknik med IEM och jontransport baserad på diffusionskoefficient och impedansmätning vid en specifik frekvens med hjälp av elektrokemisk impedansspektroskopi (EIS). När vi utforskar IEM-området har vi insett att mindre uppmärksamhet har ägnats åt sträckbar IEM, även om den kan hitta enorma applikationer inom organisk bioelektronik och kan användas i samband med många töjbara elektronikapplikationer som töjbara skärmar och energilagringsenheter. Nuvarande IEM: er saknar formbarhet och töjbarhet som kan användas för implanterbara applikationer. Med tanke på detta diskuterar vi också vår strategi för utveckling av en sträckbar IEM. Det fokuserades också på att utveckla ett hybridtillverkningsprotokoll med flexibla OEIP med mikropatronteknik och bläckstråleskrivna membran. Dessa OEIPs tillverkades och funktionaliteten validerades av cellsvaret efter leverans av ett nervblockerande medel till mösscellerna. Hittills har OEIP tillverkats av mikropatronering och arbetskrävande manuella tekniker, vilket hindrar de spirande applika- 
tionsområdena för denna goda teknik. För att ta itu med denna fråga undersöks också ett nytt tillvägagångssätt för tillverkning av OEIP: er med screentryckteknik i denna avhandling.Sammanfattningsvis kunde vi framgångsrikt utforska området för jonbytarmembran och lägga fram en ny teknik för jondetektering och sträckbara IEM för framtida applikationer. Tillverkning av OEIPs undersöktes också, vilket resulterade i utvecklingen av ett hybridtillverkningsprotokoll med bläckstråleskrivare för OEIPs och en robust helt screen tryckt OEIP med hög tillverkning Avkastning ( $>90 \%$ ) för industriell skala. 


\section{Acknowledgements}

First and foremost, I would like to thank my supervisor Magnus Berggren for giving me this amazing opportunity to work in this ever-growing group. Your scientific curiosity, brainstorming sessions on ion separation devices, and optimistic thoughts have always inspired me. I would also like to express my sincere gratitude to my co-supervisor Daniel Simon for your continuous support throughout my Ph.D. and research; also, for your patience, motivation, enthusiasm, and immense knowledge. Your guidance helped me in all the time of research and writing of this thesis. It was an amazing experience to learn from you. To Klas Tybrandt, my co-supervisor, your insightful comments, willingness to answer simple questions, and critical thinking helped me question various scientific problems and approach them from a different angle.

Besides my advisors, I would like to thank my mentors. Astrid Armgarth, for my Ph.D. kick-off. I learned a lot from you, without your initial support my Ph.D. would not have reached the heights where it is now. David Poxson, with your unique approach to science, working together with you was a wonderful experience. David Nillson for your calm project management style. Simone F, Igor Z, Eleni S, Mikhail V, Viktor G, Magnus J, Matts F, Viktor, Xavier, Mary D, Erik G, for the ongoing support and making LOE a great place to do science and research. Lars, Thomas, Anna, Meysam, Jenny helping the LOE labs, a place to work. Also, I would like to thank the Iontronix team especially, Arghya for being an amazing lab partner and friend. We have had many sleepless nights, doing late-night experiments in Stockholm, never-ending discussions on humanitarian and alien problems. Pelle and Erik, showing me the way to microfabrication, Theresia for sharing expertise on ion pump.

Friends that I scored gave life to my Ph.D. They have been there through the thick and thin. I will always be thankful for all the endless talks, crazy parties, trips, numerous cabins stays, birthday celebrations, movie nights, board games, and many more. You all have made Sweden and my $\mathrm{PhD}$ an easy ride. I know I have made friends and memories for a lifetime. Thank you, my LSD!- Lonni, Sami, and Jacky (for being there with me all through my 4 years of an eventful life. Don't know what I would do without you guys. See LSD! is covered under copyright). Special shout out to Sami for your contribution to this thesis, Eva (special mention on lending your hands for stretching my ion pumps proving your dedication to science and for all the schnapps bonding), Chiara (the very first friend I made in Sweden and I am glad to 
finish our Ph.D. journey together (fingers crossed) but never our friendship), Evelina (my eyebrows are sleek because of you ), Kaya (for the musical nights), Laura (for the good homemade foods), Donghyun (for all the never-ending talks), Nadia(for all the energy and miniman), Daniel (for the brewing sessions), Tobias (for the parties, dance, science, and universe), Jenn and fam (for all the fun time and baby Nora), Jonathan (for the concert), Lulu(for the Cerveza and fun), Hazbar (for the life hacks and talks), Tero and fam (for Finnish food and sauna).

I am also glad to have met many inspiring people from my office and outside. Yusuf for being a great college and a great mentor, Xin my office neighbor for making office time amazing and helping me with Svenska abstract. Johannes G for being a good friend and proofreading this thesis. Yiannis, Marie, Bernard, Gwynn, Johannes B, Evan, Maria, Fareed, Mehmet, Hamid, Arman, Ulrika, Cyril, Nara, Najmeh, Roman, Iwoana, Benjamin, Diana, Gabor, Rob, Roman, Jesper, Kosala, Valerio, Xeno, Vasileos, Marios, Hanne, Changbai, Daniela, Harikesh, Maciej, Mina, Asher, Aiman, Sarah, Hady, Daghmawi for the lab and outside lab times. Getting to know each one of you was great fun and memorable. I would also extend my thanks to Sci-hub for always being there for me even when the university VPN gave up when I was on the move.

I would also like to thank and dedicate this to my family for being there for me all through my life, Acha and Amma for believing in me and being proud of me always, Davis and Danis for being the best brothers and friends, Jinu chechi for being the nicest chechi and Jake an inspiring young buddy, Daddy, and mummy for being good listeners, Sanju for being the best aliyan and Nithya ready to party buddy, and aliyathi Salvi for being the cutest little sister. Finally, I would like to thank, Saumya for being my best friend and sharing your life with me, without you this book would not have been possible. 


\section{List of Publications}

\section{Publications included in the thesis}

1. Iontronic separation: fluid flow free and fully electronic ion detection Dennis Cherian, Maria Seitanidou, Klas Tybrandt, Magnus Berggren, Daniel T Simon

(under revision for resubmission)

Contribution: Idea development, device design, device fabrication, electrical and optical characterization, result evaluation, and manuscript writing.

2. Soft iontronic delivery devices based on an intrinsically stretchable ion selective membrane

Dennis Cherian, Samuel Lienemann, Tobias Abrahamsson, Nara Kim, Magnus Berggren, Daniel T Simon and Klas Tybrandt

Flexible and Printed Electronics (under review)

Contribution: Idea development, device design, material development, device fabrication, electrical and optical characterization, result evaluation, and manuscript writing .

3. Flexible organic electronic ion pump fabricated using inkjet printing and microfabrication for precision in vitro delivery of bupivacaine

Dennis Cherian, Arghyamalya Roy, Alex Bersellini Farinotti, Tobias Abrahammson, Klas Tybrandt, Camilla Svensson, Magnus Berggren, David Nilsson, David Poxson and Daniel T Simon

(manuscript ready to submit, pending submission of publication 6)

Contribution: Device design, material development, device fabrication, Electrical characterization, result evaluation (electrical characterization), and manuscript writing.

4. Large-area printed organic electronic ion pumps

Dennis Cherian, Astrid Armgarth, Valerio Beni, Ulrika Linderhed, Klas Tybrandt, David Nilsson, Daniel T Simon and Magnus Berggren

Flexible and Printed Electronics 4, 022001 (2019) 
Contribution: Development of device fabrication techniques, electrical, chemical and optical characterization, result evaluation, and manuscript writing.

\section{Related publications not included in the thesis}

6. A digital nervous system aiming toward personalized IoT healthcare Astrid Armgarth, Sandra Pantzare, Patrik Arven, Roman Lassnig, Hiroaki Jinno, Erik O. Gabrielsson, Yonatan Kifle, Dennis Cherian, Theresia Arbring Sjöström, Gautier Berthou, Jim Dowling, Takao Someya, J. Jacob Wikner, Göran Gustafsson, Daniel T. Simon and Magnus Berggren

Scientific Reports 11, 7757 (2021)

7. Electrophoretic delivery of clinically approved anesthetic drug for pain therapy

Arghyamalya Roy, Alex Bersellini Farinotti, Dennis Cherian, Tobias Abrahammson, Klas Tybrandt, Camilla Svensson, Magnus Berggren, David Nilsson, David Poxson and Daniel T Simon

(manuscript in final draft phase) 
Abstract

Populärvetenskaplig Sammanfattning iii

Acknowledgements $\quad v$

List of Publications vii

Contents $\quad$ ix

1 Introduction 1

1.1 Organic bioelectronics and iontronics . . . . . . . . . . . . . . . . 1

1.2 Thesis: outline and aims . . . . . . . . . . . . . 3

I Theoretical concepts $\quad 5$

2 Towards iontronic devices 7

2.1 Iontronic resistor or organic electronic ion pump (OEIP) . . . . . . 8

2.2 Ionic diodes . . . . . . . . . . . . . . . . . . . . . . 9

2.3 Ionic transistors . . . . . . . . . . . . . . . . . 10

3 lon exchange membranes 13

3.1 Ion conduction . . . . . . . . . . . . . . . . . . . 13

3.2 Diffusion coefficient and ion transport . . . . . . . . . . . . 14

3.3 IEM types . . . . . . . . . . . . . . . . . . . . . . . 17

3.4 IEM structure and transport mechanism . . . . . . . . . . . . . . . 18

3.4 .1 IEM microstructures . . . . . . . . . . . . . . . . . . 18

3.4 .2 Ion transport mechanism . . . . . . . . . . . . . . . 19

3.5 Permselectivity and transport number . . . . . . . . . . . . . . . 20

3.6 Influence of boundary layers in ion transport . . . . . . . . . . . 21

4 Towards flexible and stretchable devices 23

4.1 Routes to flexible and stretchable systems . . . . . . . . . . . . . . 23 
4.1.1 Softness \& low temperature processability . . . . . . . . . 23

4.2 Basic material properties . . . . . . . . . . . . . . . . 24

4.2 .1 Film failure . . . . . . . . . . . . . . . 26

4.3 Mechanics of flexible and stretchable devices . . . . . . . . . . . . . . 27

4.3.1 Mechanics of flexible mode . . . . . . . . . . . . . . 27

4.3.2 Mechanics of stretchable mode . . . . . . . . . . . . . 28

4.4 Functional electronic components and devices . . . . . . . . . . . . 28

4.4 .1 Supporting substrates . . . . . . . . . . . . . 28

4.4 .2 Functional materials . . . . . . . . . . . . . . . . . . 29

4.4.2.1 Metals .................... 29

4.4.2.2 Carbon materials . . . . . . . . . . . . . . . . . 29

4.4.2.3 Conducting polymers . . . . . . . . . . . . . 30

4.4.2.4 Hydrogels . . . . . . . . . . . . . . . . . . . 30

4.5 Pattern engineering . . . . . . . . . . . . . . . . . . . . 30

4.5.1 Precolation networks . . . . . . . . . . . . . . 30

4.5.2 Buckled structures . . . . . . . . . . . . . . . . . . 31

4.5.3 Serpentine structures . . . . . . . . . . . . . . . . . . 31

4.5.4 Helix structure . . . . . . . . . . . . . . . 32

II Experimental techniques and results 33

5 Materials and fabrication methods $\quad 35$

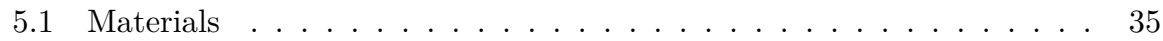

5.1 .1 Substrates . . . . . . . . . . . . . 35

5.1.1.1 Polyethylene terpthalate (PET) . . . . . . . . 35

$5.1 .1 .2 \quad$ Polyimide $(\mathrm{PI}) \ldots \ldots \ldots \ldots$

5.1.1.3 Polydimethylsiloxane (PDMS) . . . . . . . . . . 36

5.1 .2 Electrodes . . . . . . . . . . . . . . . . . 36

5.1 .3 Ion exchange membranes (IEMs) . . . . . . . . . . . . . 37

5.1 .3 .1 PEDOT:PSS . . . . . . . . . . . . 37

5.1.3.2 Hyperbranched polyglycerol (HPG) . . . . . . . . 37

5.1 .3 .3 PSS-co-MA . . . . . . . . . . . . . . . 38

5.2 Fabrication methods . . . . . . . . . . . . . . 38

5.2 .1 Screen printing . . . . . . . . . . . . . . . . . 38

5.2.1.1 Screen . . . . . . . . . . . . . . . 39

5.2 .1 .2 Squeegee .................... . . 40

5.2 .2 Inkjet printing . . . . . . . . . . . . . . . . . . . . 40

5.2 .3 Photolithography ............................. 42

5.2 .4 Physical vapor deposition (PVD) . . . . . . . . . . 44

6 Devices developed in the thesis 45

6.1 Iontronic separation . . . . . . . . . . . . . . . . . . . . . 45

6.2 Stretchable OEIP . . . . . . . . . . . . . . . . . . 47 
6.3 In vitro flexible ion pump . . . . . . . . . . . . . . . . . . . . . 48

6.4 Fully screen-printed ion pump . . . . . . . . . . . . . . . . . . 49

7 Characterization $\quad 51$

7.1 Electrical characterization . . . . . . . . . . . . . . . . 51

7.1.1 Voltage and current measurement . . . . . . . . . . . . . . 51

7.1 .2 Impedance spectroscopy (IS) . . . . . . . . . . . . . . . . . 51

7.2 Optical characterization . . . . . . . . . . . . . . . . 52

7.2 .1 Surface and rheological studies . . . . . . . . . . . 52

7.2 .2 Fluorescence microscopy . . . . . . . . . . . . . . . . . 52

7.3 Linear stretching system . . . . . . . . . . . . . . . . . 53

8 Concluding remarks $\quad 55$

Concluding remarks $\mathbf{5 5}$

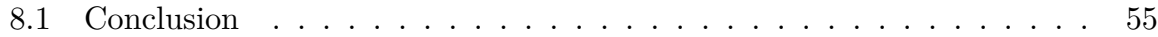

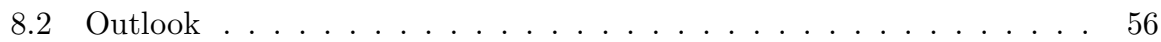

$\begin{array}{lr}\text { References } & 59\end{array}$

$\begin{array}{ll}\text { Journal articles } & 71\end{array}$

Paper I. Iontronic separation: fluid flow free and fully-electronic ion detection $\quad 73$

Paper II. Soft iontronic delivery devices based on an intrinsically stretchable ion selective membrane

Paper III. Flexible organic electronic ion pump fabricated using inkjet printing and microfabrication for precision in vitro delivery of bupivacaine 


\section{CHAPTER 1}

\section{Introduction}

The field of electronics that studies the interface between biology and electronics is called bioelectronics. This field was historically named in 1991 during the CEC workshop in Brussels and was defined at the time as the use of biological materials and biological architectures for information processing systems and new devices [1]. The first known study on bioelectronics was recorded during the 19th century when Luigi Galvani applied a voltage to a frog's leg and it twitched [2]. After Galvani, it was identified that the twitching of the frog's leg is because of ions and the charged components within the body. There has been tremendous progress in the field of bioelectronics since then and today the range of applications of bioelectronic devices spans through biosensors, drug delivery devices, implantable energy storage devices, neural implants, electrophysiological measurement devices, etc. $[3,4]$

\subsection{Organic bioelectronics and iontronics}

Electronics, in particular sensors and actuators, is generally a matured technology, while the challenges associated with bioelectronics remain in establishing a steady transmission pathway between the biological and electronic systems. Hydrogels are extensively used in bioelectronic systems as a material of interface between biological tissue and inorganic electronic materials due to their versatility [5-7]. Nevertheless, these materials are not semiconductors, and this limits their use in biological applications. Inorganic materials (i.e., "conventional electronics") have been extensively used in the field of bioelectronics because of the well- established integrated circuit industry associated with inorganic semiconductors [2]. These azoic materials have major downsides when it comes to developing a good interface with living tissue due to the mismatch between physical and chemical properties [8-11].

Communication in biological systems is generally facilitated through mixed electronic and ionic/chemical signaling. In distinction to inorganic semiconductors, organic semiconductors match with the conduction and mechanical properties of biological tissue. Because of organic electronic materials' mixed electronic and ionic conduction, they can be used as effective "bridges" for translation of biological signals to ionic signals and vice versa. For these reasons, organic semiconductors have lately been used for a broad spectrum of bioelectronics applications. For example, to more directly sense and monitor relevant physiological parameters and convert them 
to electronic signals [12], or conversely, to regulate (actuate) the physiology of cells and tissues with high spatiotemporal resolution [13]. In addition, organic semiconductors have the advantage of mechanical flexibility, the softness required for biotic interfaces, and ease of processing. Specifically, organic semiconductors can present as highly tunable soft materials with Young's modulus between $20 \mathrm{kPa}$ to $3 \mathrm{GPa}$, which is considerably closer to the biological system $(\sim 10 \mathrm{kPa})$ than inorganic semiconductors ( 100 GPa) [14-16]. The table 1.1 shows the comparison between the material properties of inorganic materials, organic materials, and living tissue. It is evident that organic electronics/conjugated polymers can be superior to the abiotic electronic or inorganic materials for bioelectronic applications [5, 17-19].

The branch of organic electronics which relies most heavily on the dual ionicelectronic capability of the organic materials is called "iontronics" [20-22]. Iontronics exploit the coupling of the electrical and ionic signals in organic electronics for use in applications ranging from electrochemical transistors, to biosensors, and most importantly, the devices that are the focus of this thesis, "iontronic transport and delivery devices". In these devices, ions are transported to a target site by an applied electric field using ionic conductors. These ionic conductors can be considered as the heart of various iontronic devices, such as the organic electronic ion pump, ionic diodes, and ionic transistors. These devices can be combined and connected to form circuits for multiplexing, signal processing, etc., and can be used for high spatiotemporal delivery or transport of ions as charge carriers rather than electrons in electrical circuits [23]. More about these iontronic materials, devices and applications will be discussed in the upcoming chapters. There have been studies on enhancement of performance and functionality of the OEIP by fine tuning the geometry of the channels for delivering neurotransmitters to nerve cells and tissue. There have been efforts made to minimize the delivery dynamics of neurotransmitters down to 100s of milliseconds. By including a preloading channel and including a waste system in a single lateral channel OEIP, OEIPs were able to achieve delivery of neurotransmitters as fast as $200 \mathrm{~ms}$ (from

\begin{tabular}{|l|l|l|l|}
\hline Parameter & $\begin{array}{l}\text { Inorganic } \\
\text { materials }\end{array}$ & $\begin{array}{l}\text { Organic electronic } \\
\text { polymers }\end{array}$ & $\begin{array}{l}\text { Biological } \\
\text { systems }\end{array}$ \\
\hline Morphology & $\begin{array}{l}\text { Crystalline } \\
\text { or amorphous }\end{array}$ & $\begin{array}{l}\text { Amorphous or } \\
\text { semicrystalline }\end{array}$ & $\begin{array}{l}\text { Cells, } \\
\text { intercellular } \\
\text { spaces }\end{array}$ \\
\hline Elasticity & $\begin{array}{l}\text { Young's modulus } \\
\sim 100 \mathrm{GPa}\end{array}$ & $\begin{array}{l}\text { Young's Modulus } \\
20 \mathrm{kPa}-2 \mathrm{MPa}\end{array}$ & $\begin{array}{l}\text { Young's Modulus } \\
\sim 10 \mathrm{kPa}\end{array}$ \\
\hline Signal carriers & Electrons and holes & $\begin{array}{l}\text { Ions, electrons, } \\
\text { and holes }\end{array}$ & Ions \\
\hline Physical state & Hard & Soft & Very soft \\
\hline Surface & Uniform & Non uniform, rough & $\begin{array}{l}\text { Dynamic and } \\
\text { complicated }\end{array}$ \\
\hline
\end{tabular}

Table 1.1. Material property comparison 
voltage-on to recorded delivery). Efforts were made to reduce the distance between the delivery point and pre-loaded channel by changing the traditional lateral delivery to vertical delivery (ion transport distance reduced to $\sim 100 \mathrm{~nm}-\sim 10 \mu \mathrm{m}$ ) [24]. This geometry further improved delivery speed to $50 \mathrm{~ms}$. As with any electronic devices, the iontronic components can be combined to form digital circuits [25-28]. Hydrogel circuits based on OEIP-derived ion junction bipolar transistors (IBJTs), have been used to demonstrate ionic NOT and NAND gates. Bipolar membrane ionic diodes have also been demonstrated for water splitting and $\mathrm{pH}$ regulating AND gates. All of these circuits share the OEIPs capability for ion transport through ion conducting materials without significant liquid transport. Many flaws have been already addressed paving the way to real life applications, for example by using palladium electrodes to trap protons for selective $\gamma$-aminobutyric acid (GABA, neurotransmitter) delivery reducing unwanted proton delivered to the target [29].

As discussed above, there has been significant advancement and effort devoted to improving the functionality and efficiency of iontronic devices. This thesis aims at understanding the ion transport and ion exchange membranes, also exploring the functionality of the above explained iontronic devices not limited to spatiotemporal delivery of biorelevant ions. Specific attention is given on process development through device design and fabrication through conventional (microfabrication through photolithography, etching, and thin-film evaporation) and unconventional technologies such as screen printing, inkjet printing, stencil, and laser patterning.

\subsection{Thesis: outline and aims}

In Paper I we discuss an analytical detection technique that can be used for detection of ions in solution. We used a simple iontronic device (OEIP) for transport of ions and the devices were designed to detect ions based on impedance spectroscopy (IS). The design, architecture and fabrication are discussed in detail. In this work we were able to successfully demonstrate the detection of inorganic and organic ions in a solution, as well as smaller inorganic ions $\mathrm{Ca}^{2+}$ and $\mathrm{Na}^{+}$which are associated with drinking water. In Paper II we explore the area of a stretchable ion exchange membrane. Here, we discuss about the development of a stretchable IEM developed with hyperbranched polyglycerol as the base membrane (a well-studied materiel for ion selective properties and transport of "larger" ions) and water-based polyurethane (WPU) as a main component. The stretchable property of the membrane is tested and established by fabricating an ion pump on a stretchable polydimethylsiloxane (PDMS) substrate. Membrane development, device fabrication, and mechanical studies are also discussed to get an insight to the stretchable properties. In Paper III we discuss the development of an inkjet ink based on hyperbranched polyglycerol and integration onto a flexible substrate using microfabrication techniques to form a lateral transport and vertical delivery OEIP system, specifically designed for in vitro studies. These OEIPs were fabricated, and the functionality was confirmed by cell response after delivery of a 
nerve-blocking agent. In Paper IV, we discuss, fully screen-printed flexible OEIPs. Ion selectivity and performance of the developed OEIPs are validated for both screen printed cation and anion exchange membranes. Small ions $\left(\mathrm{Na}^{+}, \mathrm{K}^{+}, \mathrm{Cl}^{-}\right)$as well as the biologically-relevant acetylcholine, and the anti-inflammatory salicylic acid were successfully transported and quantified both electrically and chemically.

This thesis is divided into several chapters to understand the theoretical and practical aspects of the works elaborated in the four Papers. Chapter 2 discusses iontronic devices including the OEIPs which are studied in three of the papers. Chapter 3 gives a theoretical understanding of the ion exchange membranes (IEMs) and ion conduction mechanisms. Chapter 4 introduces flexible and stretchable devices, their mechanical and functional properties, and some of the electronic components which are well exploited in our studies and devices. Chapter 5 focuses on different types of materials and fabrication techniques that are used in the thesis, all the major microfabrication methods, deposition and printing techniques are given particular importance. Chapter 6 gives a brief illustration of all the devices that were developed using the techniques mentioned in Chapter 5. Chapter 7 focuses on characterization techniques for all the devices that are described in the previous chapter. Finally, all the works presented are summarized and the scope for future works are discussed in the Conclusion. 
Part I

Theoretical concepts 



\section{CHAPTER 2 \\ Towards iontronic devices}

As mentioned in the introduction section, iontronic devices (based on the OEIP concept) are devices that utilize the dual ionic and electronic conducting properties of conducting polymers. Ions are transported to a target site by an applied electric field using ionic conductors or IEMs (ion exchange membranes). The IEMs can be polyanionic (cation exchange membranes, CEMs) or polycationic (anion exchange membranes, AEMs) which primarily conduct positive or negative charges, respectively [30]. The selectivity towards either cations or anions are based on fixed charge concentration of these IEMs. The unique characteristics of these electrophoretic devices is that the ionic transport is not fluid-flow based and hence no bulk transport occurs across these devices, only charges are transported to the target site [9].

Iontronic devices have mainly two components, electrodes and IEM, of which the IEM can be considered as the heart of these devices [31]. Several IEMs have been successfully used in various iontronic components and there has been tremendous growth in the field of IEMs for iontonic devices from linear IEM materials that show excellent conducting properties for smaller ions like $\mathrm{Na}^{+}, \mathrm{K}^{+}, \mathrm{Ca}^{2+}, \mathrm{Cl}^{-}$, and $\mathrm{I}^{-}$and biomoelecules less than $200 \mathrm{~g} / \mathrm{mol}$ like $\mathrm{GABA}^{+}$, acetylcholine $\left(\mathrm{Ach}^{+}\right)$, and salicylic acid $\left(\mathrm{SA}^{-}\right)$, to tailor-made IEM materials such as hyperbranched polyglycerol that has shown excellent proven transport properties for larger ions like indigo carmine $(466 \mathrm{~g} / \mathrm{mol})$ and methylene blue $(320 \mathrm{~g} / \mathrm{mol})$ [13,32-35]. The most widely used ion transport membrane for the first generation OEIPS was over-oxidized PEDOT:PSS acting as a CEM (see section 5.1.3.1). Later, various other IEMs like poly(styrene sulfonate)-co-maleic acid cross-linked with polyethylene glycol (PSS-coMA/PEG), poly(diallyldimethylammonium chloride) (pDADMAC), and polycationic poly (2-acrylamido-2-methyl-1-propanesulfonic acid) (pAMPSA) with their better selectivity and ease of processing and fabrication found their place in various iontronic devices [36-38]. AEMs were also developed in parallel poly(vinylbenzyl chloride) quaternized by dimethylbenzylamine (qPVBC) and poly(vinylbenzyl chloride) quaternized by triphenylphospine (PVBPPh3, which along with CEMs showed application in iontronic transistors and diodes [39] (see description below). The resistivity and size of the ions that could be transported through the IEM depends on the effective pore size and molecular dynamics of the transported species; fixed charge concentra- 
tion and pore size are the determining factors for the ion selectivity of an IEM. In addition to the specific IEM properties, the choice of IEM also depends on the charge and configuration of the molecule to be transported, compatibility with printing and micro-fabrication techniques, intended application, and substrates $[21,40]$.

\subsection{Iontronic resistor or organic electronic ion pump (OEIP)}

The OEIP makes use of polyelectrolytes of selective ion conductivity. In OEIPs these polyelectrolytes are patterned onto any substrates and encapsulated. There will be source and target reservoirs as shown in Figure 2.1 and two electrodes for source and target [13]. The polyelectrolyte connecting the source and channel electrolyte is composed of a polyanion (considering Figure $2.1 \mathrm{a}$ as an example), where the negative fixed charges on the polymer are electrostatically compensated by mobile cations. Because of the fixed anionic backbone of the polymer, anions are repelled. The high ratio of mobile cations to anions makes the channel cation selective. The ions are induced into the membrane by the application of an electric field, positive bias to the source electrolyte and negative bias to the target electrolyte. This results in cations from the source electrolyte being transported to the target electrolyte through the membrane. A small amount of water is also transported with these ions [41,42], but bulk liquid flow is avoided. These devices were proven to successfully deliver many biologically relevant ions like $\mathrm{ACh}$, GABA, glutamate (Glu), various amino acids, excitatory neurotransmitters, etc.

A basic OEIP consists of a polyelectrolyte channel, a source and target reservoir, and electrochemically active source and target electrodes. Application of electric field between source and target induces anions or cations to move depending upon the bias to the polymer. The electrodes must be capable of translating electronic signal to ionic signal. Thereby relies on the electrodes' redox capacities which is the measure of the material's available sites for oxidation or reduction at anodic or cathodic potentials, respectively $[43,44]$. If the polymer membrane is a CEM (with fixed anionic charge) it transports cations when they are introduced into the membrane and if the polymer membrane is an AEM (with fixed cationic charge) anions are transported. As an example if we consider the most commonly used PEDOT:PSS as the electrode material. When an electric field is applied across a CEM, an electrochemical circuit is established. This leads to oxidation of the source electrode and reduction at target electrode where $\mathrm{C}^{+}$is the cation present in the source electrolyte:

$$
\begin{aligned}
& \text { PEDOT }^{0}+\mathrm{C}^{+}: \mathrm{PSS}^{-} \rightarrow \mathrm{PEDOT}^{+}: \mathrm{PSS}^{-}+\mathrm{C}^{+}: \mathrm{PSS}^{-}+\mathrm{e}^{-} \\
& \text {PEDOT }^{+}: \mathrm{PSS}^{-}+\mathrm{C}^{+}: \mathrm{PSS}^{-}+\mathrm{e}^{-} \rightarrow \mathrm{PEDOT}^{0}+\mathrm{C}^{+}: \mathrm{PSS}^{-}
\end{aligned}
$$


a)

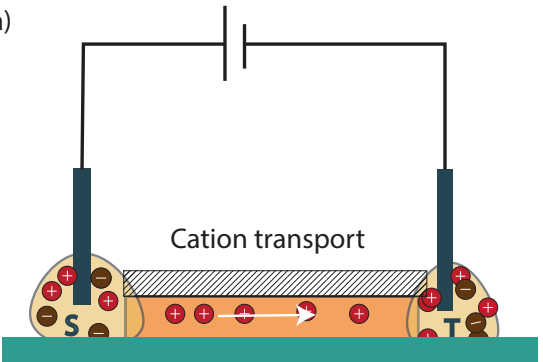

1

1

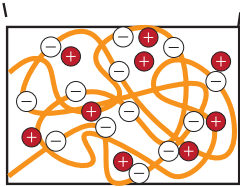

b)

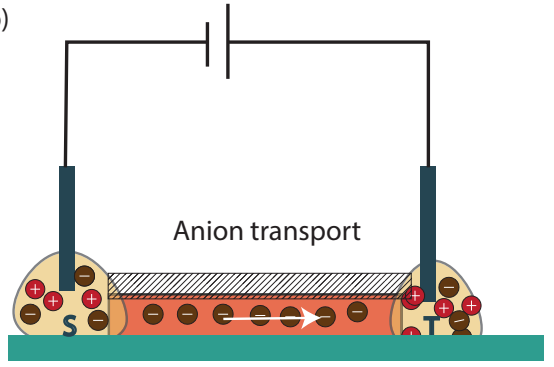

1

1

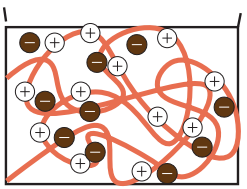

S Source

CEM

๑ Mobile positive charge $\oplus$ Fixed positive charge

T Target

Electrode

AEM

$\Theta$ Mobile negative charge $\Theta$ Fixed negative charge

Figure 2.1. An organic electronic ion pump, the white arrow indicates the direction of movement of mobile ions a, cation transport with fixed negatively charged membrane $\mathbf{b}$, anion transport with fixed positively charged membrane.

Cations are transported from the source reservoir to the target side electrophoretically. For this reason, the lifetime of an iontronic device is restricted by the availability of oxidizable PEDOT (or other electrode material) as shown in the equations.

\subsection{Ionic diodes}

A combination of polyanion and polycation can lead to devices like transistors and diodes. Two layers stacking polyanion and polycation are called bipolar membranes (BM). These can be considered as two OEIPs: one with a CEM and another with an AEM (Figure 2.2). In forward bias, the predominant charge carriers in both layers of the BM move toward the AEM or CEM junction in the middle, and they are replenished by ions from surrounding electrolytes, which drives forward current between the electrolyte on both sides. In reverse bias, the predominant charge carriers will migrate in the opposite direction away from the junction. In this case, the ions won't be replenished at the junction, which results in notably lower reverse current $[23,28,45,46]$. We can rectify this way of using ionic current in a similar way 
as electronic diodes. This can find future applications including controlled delivery of ions for bioelectronic applications.

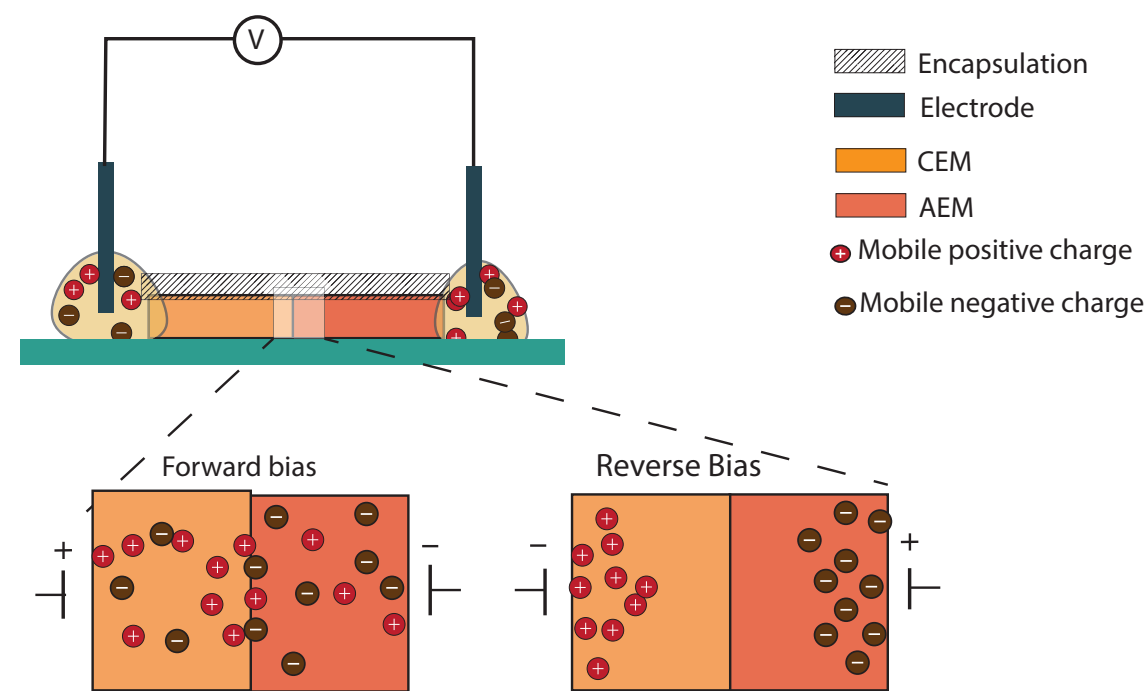

Figure 2.2. An ion bipolar membrane diode, the zoomed in image shows charged ions at the junction during forward and reverse bias.

\subsection{Ionic transistors}

Combination of two BMs or ionic diodes can lead to an ionic transistor (Figure 2.3). These are named ion bipolar junction transistors (IBJTs) and comprise three terminals: emitter, base and collector. The configuration of IBJTs is that emitter and collector are of the same polarity, meaning IBJTs are either npn or pnp. In iontronic terms npn is AEM based emitter and collector and CEM based base, pnp corresponds to CEM based emitter and collector and AEM base. $V_{E C}$ is the voltage applied to drive ions between emitter and collector and $V_{E B}$ (the voltage on the base) is used to control the emitter-collector current. Predominantly, there are two kinds of operation for IBJTs: for pnp-IBJTs the active mode is when $V_{E C}$ and $V_{E B}$ are both negative as this pushes anions from emitter and cations from the base towards the junction like a forward bias. This increased concentration allows anions to cross the base and enter the collector due to the negative bias of $V_{E C}$. When $V_{E B}$ is reversed, the transistor works in a reverse bias. This leads to ion depletion at the junction as the cation concentration is low in the base and the ionic current gets reduced and when the junction is completely depleted and no ions pass through it $[27,47]$. 


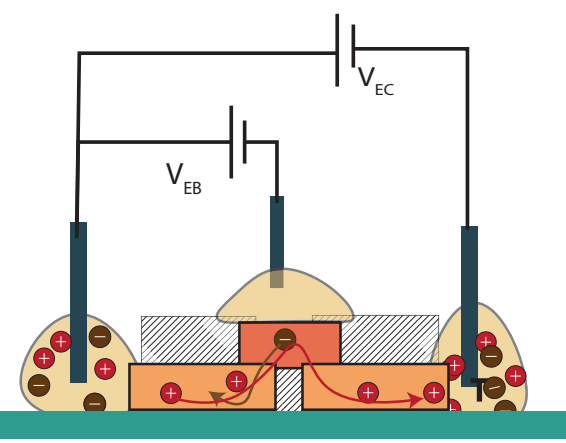

Encapsulation

\section{Electrode}

$\square$ CEM

$\square$ AEM

๑ Mobile positive charge

@Mobile negative charge

Figure 2.3. A pnp-type ion bipolar junction transistor. 


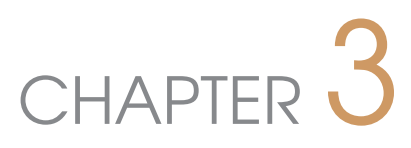

\section{Ion exchange membranes}

Ion exchange membranes (IEMs) are a class of polymeric membranes that has been extensively used in various kind of applications including electrodialysis, diffusion dialysis, flow batteries, capacitive deionization, microbial fuel cells, and ion exchange bioreactors [48-50]. Recently, they also found interesting application within the field of iontronic bioelectronic devices [13], as explained in the previous chapter. IEMs are dense polymeric membranes with fixed charges in their matrix, which allows them to selectively transport oppositely charged ions (counterions) to the fixed charges and restrict the transport of ions of the same charge (coions). The ion selectivity towards counterions in IEMs is due to an electrostatic effect called Donnan exclusion [51]. As the devices and studies in this thesis relies on ion exchange membranes and ion transport, this chapter will cover the basics of ion transport, IEMs, and selectivity.

\subsection{Ion conduction}

Electric current is the result of directional movement of ions or electrons. Conductors are divided into electronic conductors and ionic/electrolytic conductors depending on the charge carriers. Electrolytes are ionic conductors that contain mobile ions, often in the form of a dissolved salt in a solvent. Electrolytes can be subdivided into strong and weak electrolytes, where a strong electrolyte is a formed by complete dissociation of ions in the solvent while a weak electrolyte only partially dissociates. For a dissociated fixed salt concentration the conductivity tends to be higher for strong electrolytes than weak ones. The conductivity $(\sigma)$ depends on the concentration of dissociated ions in the electrolyte and their mobility $\left(\mu_{i}\right)$.

$$
\sigma=\sum_{i} z_{i} \mu_{i} v_{i} c F
$$

where $z_{i}, \mu_{i}, v_{i}$ are the charge number, mobility, and stoichiometric coefficient of ion $i$, respectively. $c$ is the electrolyte concentration, and $\mathrm{F}$ is the Faraday constant. An interesting phenomenon is that for high concentrations, the ionic conductivity can 
decrease due to inter ionic forces [53]. This highlights the complexity of ionic transport where quantities like mobilities change with respect to concentration and other environmental factors. Ionic conductivities are typically much lower than conductivities in electronic conductors, as the movement of ions is slow in comparison to the electrons [52]. For instance, typical ionic conductivities are less than $100 \mathrm{~S} / \mathrm{m}$ while metallic electronic conductivities are in the order of $10^{6}-10^{8} \mathrm{~S} / \mathrm{m}[53]$.

\subsection{Diffusion coefficient and ion transport}

An electrolyte can be defined as a mixture of cations and anions dissolved in water. In aqueous electrolyte, ion are transported based on 3 mechanisms:

\section{Diffusion,}

2. Electromigration,

3. Convection.

Diffusion can be described as the arbitrary movement of species in the space due to concentration gradient and is a result of thermal motions between particles [54,55]. The diffusion coefficient is a parameter that relates to how fast ions flow in a concentration gradient. Diffusion coefficient (D) is dependent on the size and frequency of movement of ions.

The simplest portrayal of diffusion is given by Ficks first law [56]. The rate of transfer of molecules or in other words molar flux is proportional to concentration gradient and is given by the equation:

$$
N_{i}=-D_{i} \nabla C_{i}
$$

where for species i, $N_{i}$ is the molar flux $\left(\mathrm{mol}^{-1} \mathrm{~m}^{-2} \mathrm{~s}^{-1}\right), D_{i}$ is the diffusion coefficient $\left(m^{2} s^{-1}\right)$, and $C_{i}$ is the concentration $\left(\mathrm{mol}^{-1} \mathrm{~m}^{3}\right)$.

Diffusion coefficient generally scales with the molecular size of the ions implying that smaller ions diffuses faster than larger ions. The Einstein-Stokes relation describes the relationship between diffusion coefficient and radius of the ions.

$$
D_{i h}=\frac{R T}{6 \pi \eta_{h} r N_{a}}
$$

where $\eta_{h}$ and $r$, are viscosity of the (homogeneous) phase and the radius of the (spherical) particle respectively, $R$ is the universal gas constant, $T$ is the temperature, and $N_{a}$ is Avogadro's constant. The diffusion coefficients for some of the ions are tabulated in Table 3.1. 


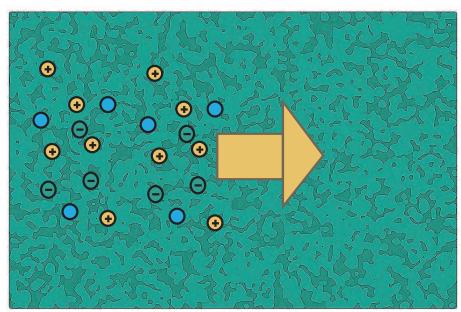

Figure 3.1. Ions moves from a region of higher concentration to a region of lower concentration, the arrow indicates the diffusion direction.

\begin{tabular}{|l|c|}
\hline Ions & $\begin{array}{c}\text { Diffusion coefficient } \\
\left(10^{-9} \mathrm{~m}^{2} \mathrm{~s}^{-1}\right)\end{array}$ \\
\hline $\mathrm{H}^{+}$ & 9.310 \\
$\mathrm{~K}^{+}$ & 1.960 \\
$\mathrm{Li}^{+}$ & 1.030 \\
$\mathrm{OH}^{-}$ & 5.270 \\
$\mathrm{Cl}^{-}$ & 2.030 \\
$\mathrm{~F}^{-}$ & 1.460 \\
\hline
\end{tabular}

Table 3.1. Diffusion coefficients of some common cations and anions.

Migration is the movement of charged molecules by external electric potential. As seen in Figure 3.2 cations migrate to the negative terminal and anions to the positive terminal. This controlled transport of ions under applied electric field is the major transport mechanism utilized in iontronic devices. The ability of the ions to be transported under applied electric field is called mobility $(\mu)$ and is related with the diffusion coefficient (D) as follows:

$$
\mu=\frac{D}{k_{B} T}
$$

where $k_{B}$ is the Boltzmans constant, and $\mathrm{T}$ is the temperature in Kelvin (K).

Transport of ions due to migration can give rise to concentration gradients and this can result in the transportation of ions due to diffusion. Similarly, diffusion can also induce electric fields The ionic flux (j) combining the transport due to diffusion and migration can be described by Nernst Planck equation:

$$
j=-D_{i} \nabla C_{i}+z D_{i} C_{i} f E
$$

where $D_{i}$ is the diffusion coefficient of the ion and $C_{i}$ its concentration. $\mathrm{f}$ is $\frac{F}{R T}$, where $\mathrm{F}$ is the Faradays constant, $\mathrm{R}$ is the ideal gas constant, $\mathrm{T}$ is the temperature in $\mathrm{K}($ Kelvin), $\mathrm{z}$ is the charge number of the ion and $E$ is the electric field. Here, the 
first term represents the flux due to ionic diffusion and the second term represents the flux due to ionic migration.

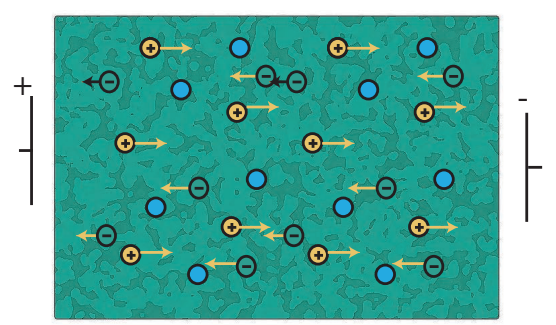

Figure 3.2. Electromigration: anions are attracted by the positive terminal and cations by the negative terminal, this initiates the ion transport to the respective terminal when subjected to external electric field.

Convection is the movement of ions due to the motion of solvents. Convection is utilized in transport of ions for longer range, hence these are widely used in microfluidic drug transport and even in iontronic delivery devices. Convection can be driven by external pressure gradients or a natural convection as a result of density gradients.

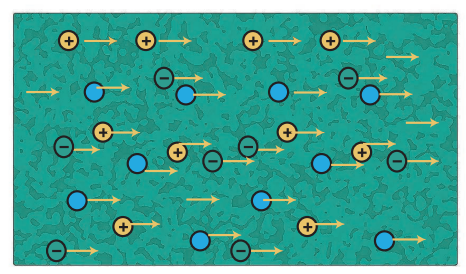

Figure 3.3. Convective transport: ionic movement in the direction of solvent motion.

The extended Nernst planck equation includes the convective term to incorporate its contribution to the the ionic transport [51]. It is defined as follows:

$$
j_{i}=v C_{i}-D_{i} \frac{d C_{i}}{d x}-\frac{z_{i} C_{i} F D_{i}}{R T} \frac{d \phi}{d x}
$$

where $v$ is the convective velocity, $z_{i}, D_{i}, C_{i}$ are the valence, diffusion coefficient and concentration respectively for the ion $i$. Here the first term represents transport due to convection, second term represents diffusion due to concentration gradient and the third one is the transport due to electromigration. 


\subsection{IEM types}

The main class of IEMs discussed in this thesis are anion exchange membranes (AEMs) and cation exchange membranes (CEMs) [51,57]. IEMs typically contain hydrophilic

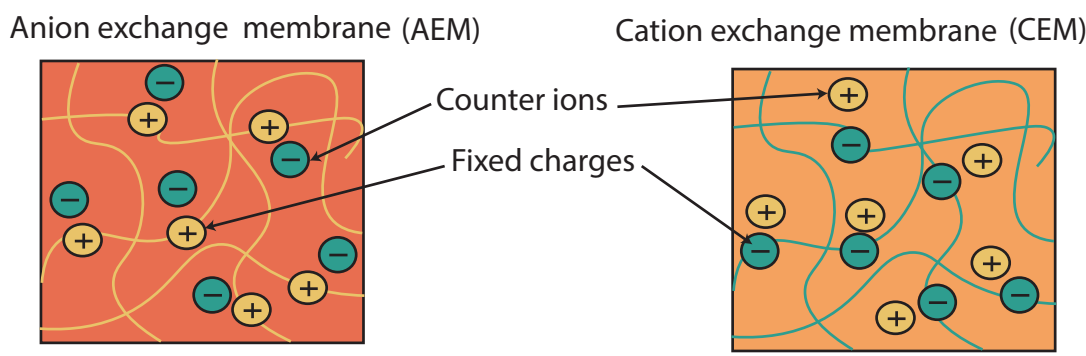

Figure 3.4. An example of AEM and CEM. The AEM has a fixed cationic group represented by yellow lines, similarly the CEM has fixed anionic group represented by green lines.

ionic groups and hydrophobic polymer backbones. When an IEM is brought in contact with water, a microphase separation can occur between the hydrophilic ionic groups and the hydrophobic polymer chains, where the hydrophilic domain accounts for the ionic transport while the hydrophobic domain provides structural stability. The ion selectivity can be explained with the Donnan effect, which is derived by considering the thermodynamic equilibrium of an IEM with homogeneously distributed fixed charges in contact with electrolytes. An electric potential is generated at the membrane solution interface (Donnan potential), which is responsible for the exclusion of coions from the IEM. The Donnan potential is mathematically expressed as [58]:

$$
\phi_{D o n}=\phi_{m}-\phi_{s}=\frac{R T}{Z_{i} F} \ln \frac{c_{s}}{c_{m}}
$$

where $\phi_{D o n}$ is the Donnan potential, $\phi_{m}$ the membrane potential, $\phi_{s}$ the solution potential, $R$ the universal gas constant, $T$ the absolute temperature, $F$ the Faraday constant, $z$ the charge, and $c_{s}$ and $c_{m}$ the electrolyte concentration of the counter ion and fixed charges in the membrane respectively. The Donnan potential, and the coion exclusion, thus increase with fixed charge density and decrease with electrolyte concentration. In a CEM the cations are attracted and anions are repelled, while for AEM the anions are attracted and cations are repelled. 


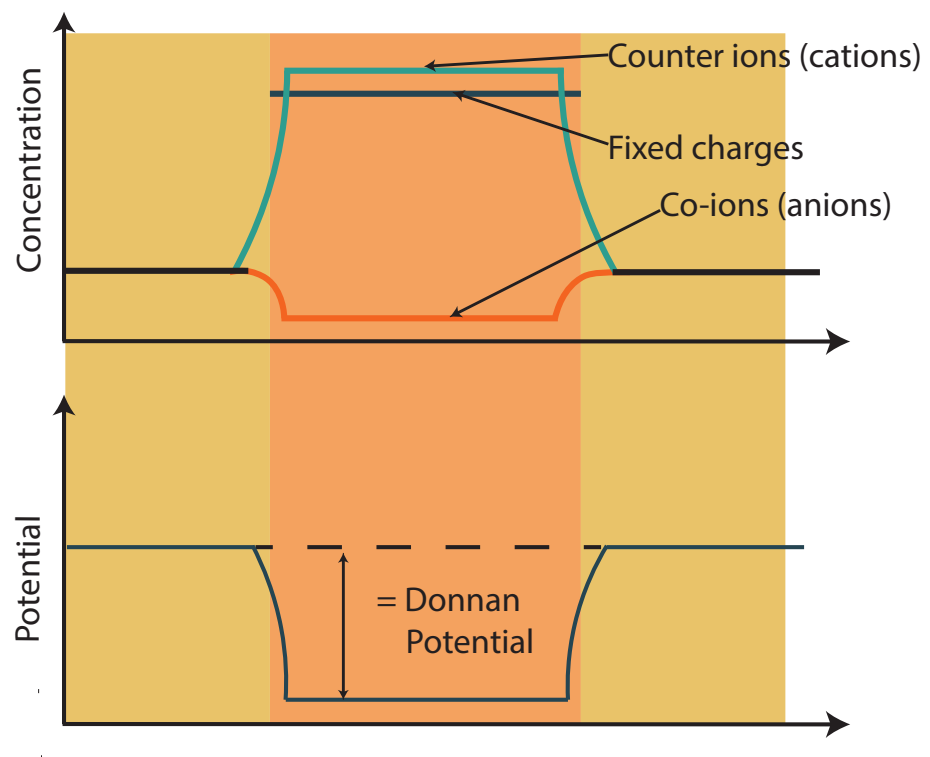

Figure 3.5. Concentration and potential changes across a CEM (orange part implies the membrane and the yellow area implies electrolytes). The diffusion of charges creates surplus of negative charges inside the membrane and positive charges outside the membrane which creates an electric field at the interface. Through the CEM, cations are attracted and anions are repelled, as the potential in the membrane is lower than the electrolyte. This potential difference is called Donnan potential [59].

\subsection{IEM structure and transport mechanism}

\subsubsection{IEM microstructures}

Ion selectivity can be understood and explained in terms of ion exchange and ion mobility in the membranes [30]. Hitherto there has been enough evidence suggesting that the adaptive microstructure of the membranes is also important while considering the ion selectivity and transport. Three different models of the membrane microstructure [60] can be used to describe the ion transport:

1. Membranes considered as a homogeneous solution

2. Membranes considered with structural in-homogeneity

3. Microphase scale 
The first model is based on the Donnan effect and IEMs are considered as a homogeneous polymer matrix with fixed and mobile ions and water. The second model assumes structural in-homogeneities of various levels in the IEMs. Here the IEMs are treated as a channel bundle or a pseudo-homogeneous solution with nonuniform fixed charges. The third model considers in-homogeneity on the microscopic scale [61]. This model considers a 2 phase system as shown in the Figure 3.6, gel phase (white area) and interstitial phase (colored area with patterns). The gel phase contains polymer chains bounded to hydrophilic ion exchange groups. Interstitial phase are the spaces/voids between gel phase [62]. These voids are created as a result of pores and structural defects and these are assumed to be filled with electroneutral solutions. These interstitial phase is responsible for ion transport when the membranes are hydrated with salt solution. The void size is not membrane specific and can vary depending on the hydration and the swelling.
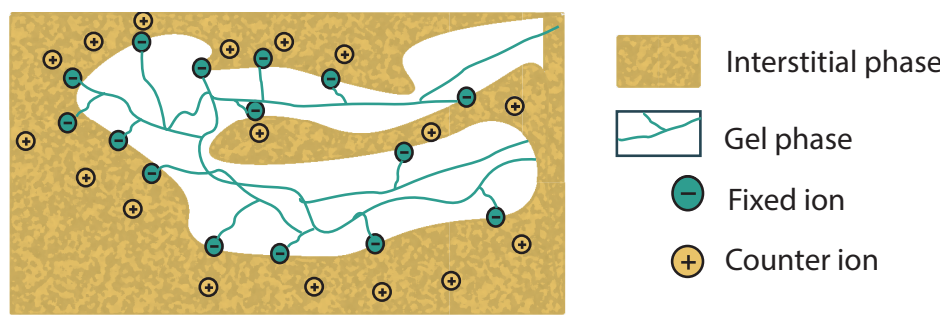

Figure 3.6. 2 phase structural model.

\subsubsection{Ion transport mechanism}

In addition to the normal transport mechanism observed in a bulk solution as described in Section 3.2, that's transport due to diffusion, convection and electromigration $(3.2[63,64])$, transport in IEMs can also be due to surface hopping. As IEMs are dense, transport due to convection is typically minimal in comparison to the transport from migration once a voltage is applied. Also, within the bulk of IEMs migration typically dominates diffusion. The extended Nernst Planck equation does not cover the ion transport due to surface site hopping. Surface site hopping as the name suggests is the hopping of counter ion from one fixed site to neighbhour site. This is the movement of ions through successive hops, from one side of the chain to the other by the electrostatic force created by the counter ions. The surface site hopping of cations through a CEM is illustrated in the Figure 3.7 as an example. Here a cation attached to fixed anionic charge is solvated and dissociated by water molecules. Bordering side anionic fixed charge attracts the cations to its surface and the process repeats. In the presence of water, the fixed ionic group in the interface of the membrane get ionized. The effective dielectric constant $(\epsilon)$ in the ion exchange membrane is lower than in the bulk solution, as a result the coulombic interaction is stronger in the exchange 
membrane phase than the bulk solution. The mobile and the ionized fixed ions are surrounded by water (forming a hydration shell). The hydration shell around the fixed charges weakens coulombic interaction between fixed and mobile ions. Thus interaction between counter ion and fixed charges are influenced by coulombic forces and the hydration of the ions. Hence, the ion transport depends on the hydrophobicity of the fixed charges and hydration energy of the mobile ions. The surface hopping also dependent on the distance between neighboring fixed charges.

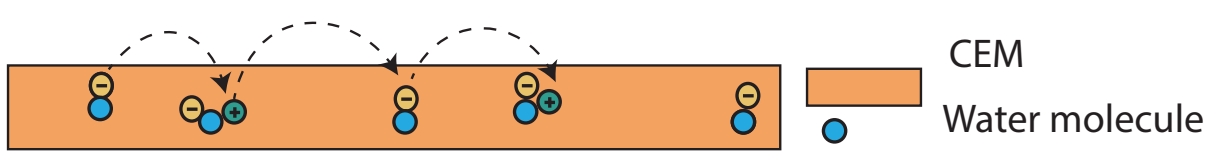

Figure 3.7. Counter-ion surface site hopping mechanism in a CEM, the arrow indicates the cation transport through hopping.

In contrast to the normal transport mechanisms mentioned above, it is worth mentioning the unusual transport mechanism of $\mathrm{H}^{+}$and $\mathrm{OH}^{-}$in solution. In solution, $H^{+}$is transported by the Grotthuss mechanism [65], where $H^{+}$ions are mainly transported from a hydronium ion to another water molecule by the reconstruction of hydrogen bonds. Here only a small amount of $\mathrm{H}^{+}$ions are transported with a hydration shell. At the interstitial of IEMs, the structural or simple diffusion is dependent on the hydration of the IEM. Higher the hydration level, more prominent is the structural diffusion and lower the hydration level simple diffusion become more prominent. In case of $\mathrm{OH}^{-}$, the transportation is best described by hyper coordination theory. A hydroxide ion forms a square planar topology in water, the oxygen accepts 4 hydrogen atoms from neighboring water molecule. The hydrogen atom of hydroxide is delocalized around oxygen and stays without bonds. For charge defect transport to happen, hydroxide ions reduces its coordination number by breaking one of its hydrogen bonds received from water molecule, and then establishes the bond with the neighboring water molecule. This transfers the defects to the neighboring molecule. At the end of the transfer, molecule rearranges to form a square planar configuration [66].

\subsection{Permselectivity and transport number}

In an electrolyte, different ions contribute differently to the ionic conductivity due to the difference in mobility as described in the diffusion coefficient section. The fraction of an ionic current resulting from transport of an ionic species i is called the transport number $\left(t_{i}\right)$. The sum of all transport numbers for the ionic species in an electrolyte is by definition 1 . In many cases the transport number depends on factors like temperature and concentration. In the case of IEMs, the concentration of coions and counter ions in the membrane can vary which influence ion transport. 
The permselectivity is the term used to define the transport selectivity of specific ions through ion exchange membranes $[67,68]$. It is defined as:

$$
P=\frac{t_{i m}-t_{i}}{1-t_{i}}
$$

where $t_{i m}$ and $t_{i}$ are the transport number of the counter ions in the membrane and the electrolyte, respectively. If all the ionic current is due to counter ions, the permselectivity value will be 1 , indicating that the membrane is perfectly permselective. In addition to the counterion/coin concentrations, other factors like boundary layers also influence permselectivity. Ions with larger diffusivity gain selectivity with an increase in boundary layer thickness.

\subsection{Influence of boundary layers in ion transport}

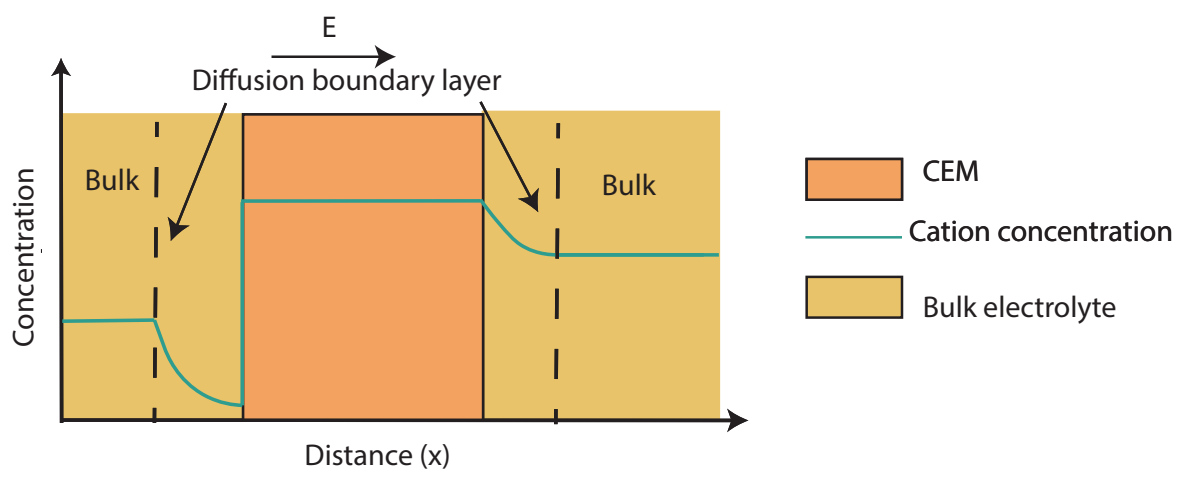

Figure 3.8. Cation concentration across a CEM.

As predicted by the Donnan effect, the coion transport increase with increasing electrolyte concentration. By applying an electric field across the membrane, the local solution concentration at the boundary layers can be highly influenced by the induced ionic current. At the side of the membrane where counterions enter the membrane, the local concentration is depleted. When increasing the current, eventually the concentration next to the membrane will approach zero. This condition limits the current which can be passed through the membrane and is called limiting current. An example of a concentration profile of cations across a cation exchange membrane is plotted in Figure 3.8 [69]. 


\section{CHAPTER 4}

\section{Towards flexible and stretchable devices}

In recent years there has been significant interest in flexible and stretchable electronics due to promising applications related to displays, healthcare, wearables, biomedical implants, and the internet of things $[60,70,71]$. The work presented in this thesis addresses the challenges of developing flexible and stretchable iontronic devices. Organic semiconductors can be soft and withstand substantial deformation, and are therefore attractive for flexible and stretchable electronics devices [18]. Because of these characteristics, organic semiconductors can be used to create a wide range of unconventional devices with form factors that are not achievable by inorganic semiconductors. The mixed ionic electronic conductivity of certain organic semiconducting materials enables their use in iontronic devices like ion pumps and ionic diodes $[21,23]$. The development of flexible and stretchable iontronic devices would allow for the technology to be integrated into existing stretchable and flexible electronic platforms.

\subsection{Routes to flexible and stretchable systems}

\subsubsection{Softness \& low temperature processability}

There are several mechanisms that are responsible for dissipation of mechanical stress from the structural complexity of a polymeric film [72]. Although the chain matrix, morphology, and packing style take part in reducing the mechanical stress, studies have shown that the weak vander waals interactions between the molecule is the main reason for the softness and deformability of these materials. Inorganic semiconductors are kept together by strong covalent or ionic bonds, which restricts their deformability. The weaker interactions within OSCs can allow the polymer chains to move with respect to each other, thereby inducing deformability within the material. Young's modulus is defined as the ratio of tensile stress to strain and describe how easily a material can be deformed, i.e. its softness. Silicon has a Young's modulus of $130 \mathrm{GPa}$, while many OSCs have Young's modulus in the GPa $[73,74]$ and some composites even below $10 \mathrm{MPa}$ [75]. 
OSCs can be deposited by thermal evaporation or from solution by a wide range of deposition techniques including various forms of printing. Some films require thermal treatments, yet the maximum temperature is often less than $200^{\circ} \mathrm{C}$. The weak bonds within OSCs makes it possible to process them under low temperatures. For a final device to be flexible or stretchable, not only the functional part but also supporting parts needs to be flexible and stretchable. For instance, the substrate and encapsulation must also have those mechanical characteristics. Low temperature processibility is relevant in this case for the use of substrates like plastics, papers and textiles [72].

\subsection{Basic material properties}

To understand the mechanics of flexible and stretchable systems, basic parameters are outlined. Stress is a measure of the internal forces that particles exert on each other within a material $\left(N / m^{2}=P a\right)$. The source of stress is mainly externally applied load but can also be due to internal mechanisms like thermal effects, variation in composition etc. $[72,76]$.

The mathematical equations representing the tension or compression stress $(\sigma)$, and shear stresses $(\tau)$ are shown below:

$$
\begin{gathered}
\sigma=\frac{F_{\uparrow}}{A} \\
\tau=\frac{F_{\leftarrow}}{A}
\end{gathered}
$$

Where $F_{\uparrow}$ is the the force applied perpendicular to the area $A$ or parallel to the axis. $F_{\leftarrow}$ indicate the force applied parallel to the area $A$ (Figure 4.1).

Strain is defined as the change in length per unit length. A strain can be due to tensile deformation, compressive deformation, and shear deformation [77]. Tensile deformation is the variation in displacement with respect to reference dimension, compressive deformation is related to compact shape and shear strain describes angular displacements. The mathematical equation representing compressive strain is:

$$
\epsilon=\frac{\delta y}{y}
$$

where $\delta y$ is the length between the final length and the origin length y.

For angles and for small displacements, shear strain is defined as :

$$
\gamma=\tan \theta=\frac{\delta x}{y}
$$

Different type of stress and strain mentioned above could be summarized as in Figure 4.1. Mechanical behavior of the materials can be described using stress strain relationship. This include but not limited to - Young's modulus, Poisson's ratio, and thermal expansion coefficient. 


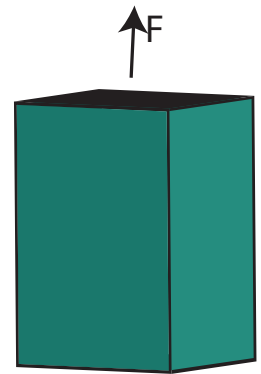

$\mathfrak{I}^{F}$

Tension

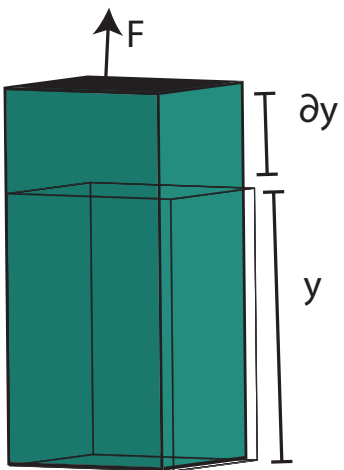

Tensile deformation

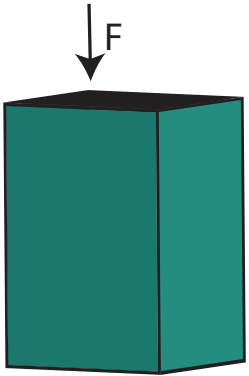

个F

Compression

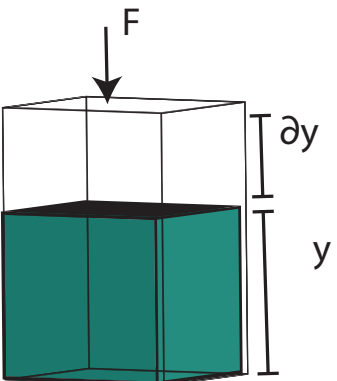

Compressive deformation
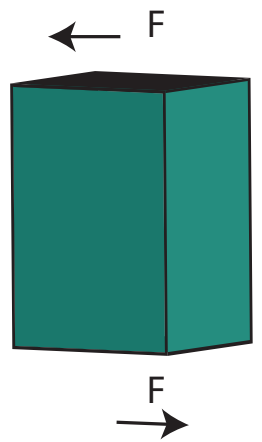

Shear stress

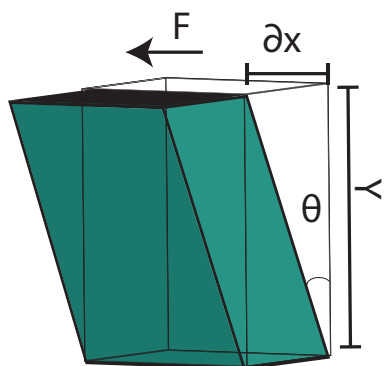

Shear deformation

Figure 4.1. Type of stress (top row) and strain (bottom row).

A material is said to be a linear elastic material if the stress depends linearly on the strain. Elasticity infers that the material will return to its original state after the load is removed, while plasticity implies the material will not return to its normal state after the load is removed. Upon increasing the load, the stress increases until it reaches a maximum point, after which it enters a necking stage. After this stage the material will not return to its original state, meaning an irreversible deformation has happened.

For a unidirectional load, the stress can be proportional to the strain, where the proportionality constant is called Young's modulus $(E)$. A high Young's modulus indicates a high stiffness, i.e. a high resistance to undergo deformation in response 
to applied force [78].

$$
E=\frac{\sigma}{\epsilon}
$$

If a body is exposed to a strain in one direction, it will also show a strain in the perpendicular direction, typically by a reduction in dimension for tensile strain. The negative ratio of transverse strain to axial strain is called Poisson's ratio $(\nu)$. Usually, the Poisson's ratio is smaller than 0.5 for most materials. If its value is 0.5 , the volume of the material will not change upon deformation, in short, the material is incompressible [72].

In multilayered devices, the residual stress is caused by the difference of thermal and humidity coefficient between the substrate and the film deposited. As there is a difference in the mechanical properties of film and a substrate, the mechanics depends on the Youngs modulus and thickness of both substrate and the deposited film.

When $E_{f} h_{f} \ll E_{s} h_{s}$ (where $E_{f}, h_{f}$ and $E_{s}, h_{s}$ are the Young's modulus and thickness of the film and the substrate respectively), the stress in the film is higher than that in substrate which can result in the structure to slightly curve in a spherical cap. Then this radius of curvature [79] and the residual stress is related according to Stoney's formula [80]

$$
\sigma=\frac{E_{s} h_{s}^{2}}{6\left(1-v_{s}\right) h_{f}}\left(\frac{1}{R}-\frac{1}{R_{0}}\right)
$$

Where $R$ and $R_{o}$ are the radius of curvature of the substrate before and after deposition. $v_{s}$ is the Poisson's ratio of the substrate. When $E_{f} h_{f} \approx E_{s} h_{s}$, the strength of the film and substrate is equal, in this case instead of forming a spherical cap it rolls into a cylinder.

\subsubsection{Film failure}

Limitations of flexible electronics system includes mechanical failure under larger deformation. Crack formation followed by propagation and interfacial delamination are the two main mechanisms that the devices fail under mechanical deformation. When a tensile strain is applied to a multilayered film, there can be failures because of parallel channeling cracks, which are caused by brittle or stiffer films deposited on the substrate [81]. More loading can lead to delamination of the film/substrate. The cracks can also channel through the film and the substrates. The possible failure mechanisms of a multilayered film is summarized in Figure $4.2[82,83]$. 
a)

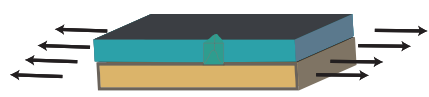

d)

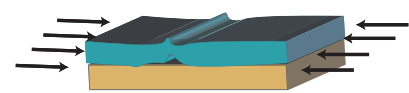

b)

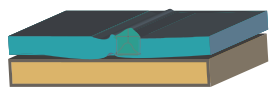

c)

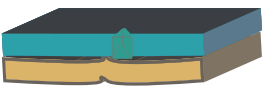

e)

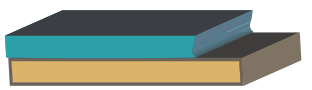

Figure 4.2. Illustration of film and substrate failure under tensile stress: $\mathbf{a}$, cracked film, $\mathbf{b}$, cracked and delaminated film, and c, cracked and substrate failure. And under compressive stress: d, buckle delamination and $\mathbf{e}$, edge delamination.

\subsection{Mechanics of flexible and stretchable devices}

\subsubsection{Mechanics of flexible mode}

In bending two sides of the flexed sheet experience two type of strains, the top of the sheet will undergo lengthening and the bottom substrate or layer undergo shortening [84]. This means that the top film will have positive strain or tensile strain and the bottom one will have negative strain or compressive strain (illustrated in Figure 4.3). This strain may lead to different kinds of failure modes, the components under tensions lead to cracks and the components under compression could lead to delamination. While bending the greater strain happens in the surface of a homogeneous substrate, it is defined as peak strain $\left(\epsilon_{\text {peak }}\right)$.

$$
\epsilon_{\text {peak }}=t / 2 r
$$

As observed from the equation, at a given bending radius(r), thinner substrates will experience lower critical strain (t represents the thickness of the film). For a flexed film, the strain varies between compressive and tensile strain maxima on either surface. This variation is different for homogeneous and layered structures. For both cases a neutral plane exists which does not have a compressive or tensile stress. Positioning devices or materials along this neutral plane can maximize the flexibility of the system. This can be done either by using materials with similar youngs modulus and thickness or by distinct materials with different thickness. then the materials will lie in the neutral plane if:

$$
E_{s} t_{s}^{2}=E_{0} t_{0}^{2}
$$




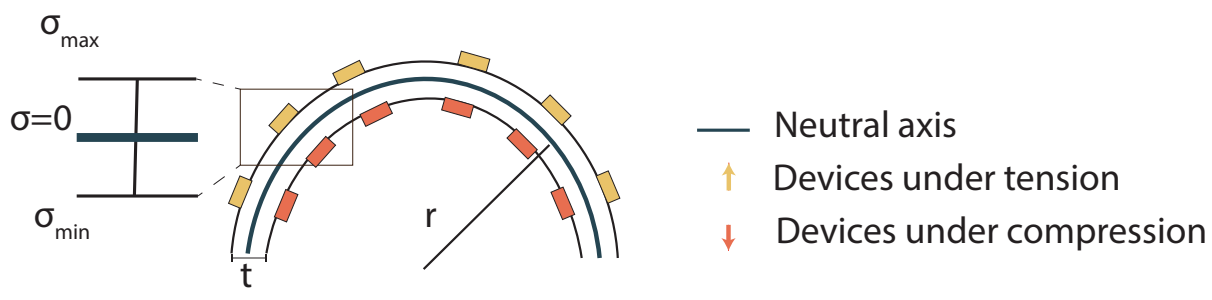

Figure 4.3. Schematics of a devices on a substrate, showing compressive and tensile stress while being flexed [72].

where $E_{s}$ and $t_{s}$ are the modulus and thickness of the substrate, and $E_{0}$ and $t_{0}$ are the modulus and thickness of the overlayer.

\subsubsection{Mechanics of stretchable mode}

Flexibility is mainly achieved through thin films and placement of sensitive components in the neutral plane. For flexible devices the critical strain is often less than $2 \%$. However, a critical strain of $2 \%$ is not sufficient for a stretchable device [85], thus, alternative approaches must be pursued for stretchable devices. As explained below, it could be through developing intrinsically stretchable materials or through pattern engineering.

\subsection{Functional electronic components and devices}

Specialized materials and structures are exploited to make a device flexible and stretchable. They can be classified into:

1. Supporting substrates,

2. Functional materials.

\subsubsection{Supporting substrates}

Polymer materials are widely used for flexible applications due to their relatively low mechanical stiffness, and good thermal and chemical stability. The most commonly used flexible substrates are polyimide (PI), polyethylene terephthalate (PET), and polyethylene naphthalate (PEN). Paper is also widely investigated as substrate material for flexible electronic applications. Because of their relatively high elastic modulus and low fracture strain, these materials are less applicable in stretchable applications. Elastomers and textiles are the main categories of materials used in stretchable electronics. Silicone based elastomers, like PDMS and Ecoflex, are widely 
used in stretchable applications, mainly because of their good processibility and chemical durability. As these materials are often cross linked with cross linking agents, the mechanical properties can be easily tuned by adjusting the amount of the cross linking agent and reaction conditions [86]. The surface properties of these materials can also be easily tuned to enhance the adhesion of functional materials to the substrate $[87,88]$. Textiles are interesting for stretchable substrates due to their $2 \mathrm{D}$ planar structure, with intervened fibers, which is good at adsorbing functional materials and delivering deformability [89].

\subsubsection{Functional materials}

The electronic or ion conductivity should be maintained after the functional material deposition. Major types of functional materials that are being investigated for stretchable and flexible applications are as follows:

- Metals,

- Carbon materials,

- Conducting polymers,

- Hydrogels.

\subsubsection{Metals}

There has been a lot of studies developing metal nanoparticles and nanowires for stretchable electronics applications. Silver, gold and copper-based nanomaterials have been proven as appropriate conductive materials for stretchable electronics [90-92]. These nanomaterials are processed with different solvents and additives and are transferred to the substrates by printing or other compatible deposition processes. In some cases these nanomaterials were made into composites by mixing them with stretchable materials like polyurethane, PDMS, etc [93].

\subsubsection{Carbon materials}

The most commonly used carbon-based materials include carbon black, carbon nanotubes (CNTs), and graphene. CNTs are made by rolled up single layer atoms, forming nano sized cylindrical cores [94,95]. These materials are known for their high elastic modulus and low cost, and are demonstrated as efficient materials for stretchable and flexible conductors. These materials have been successfully used in stretchable electronic devices along with PDMS, SEBS, and fluro elastomers as substrates [96]. Graphene and graphene-based composites have also been proven to be a good candidate for stretchable conductors. 


\subsubsection{Conducting polymers}

Conducting polymers are organic polymers that can conduct electricity. Though the conductivity of these polymers are typically much lower than in metals, they possess good compatibility with flexible and stretchable substrates. PEDOT:PSS is one of the most studied CPs, and PEDOT:PSS has been successfully demonstrated on various flexible substrate intended for wide range of applications. Solution processed PEDOT:PSS mixed with ionic liquids deposited on SEBS, PEDOT PSS composite with polyurethane hydrogels are some examples of PEDOT:PSS based stretchable devices or conductors [97-99]. There have been reports demonstrating stretchable actuators based on other conducting polymers like PPy, PANi etc., due to their property to induce strain to the applied voltage. These actuators could operate under low voltage $(<3 \mathrm{~V})$, provide high strain from 1-40\% and relatively high stress $(\sim 35 \mathrm{MPa})$ [100].

\subsubsection{Hydrogels}

Hydrogels are polymer network infiltrated in water, which are usually brittle in nature [101]. Tough hydrogels are the class of hydrogels with extraordinary mechanical properties. Many tough hydrogel based stretchable electronics has been recently reported with high water content (75-95\%), high mechanical robustness, stretchability and bio compatibility [102]. These hydrogels based stretchable materials provide soft and wet matrix for electronics and devices making it suitable for stretchable electronics applications. Hydrogels of conducting polymer like PEDOT:PSS has also been studied for electrical interface with tissues for sensing and stimulation [103]. In this thesis we use ion conducting hydrogels for use in iontronic devices. Due to the unique properties, hydrogels are being investigated in wide range of stretchable and flexible applications including artificial tissues, drug delivery, and brain implants.

\subsection{Pattern engineering}

Stretchability can be achieved using different pattern engineering techniques, most commonly used approaches are described in short here:

\subsubsection{Precolation networks}

Percolation networks are widely used because of the ease of processability and low complexity. They are formed by mixing the functional material with an elastic material or dispersing the functional material on a stretchable substrate (figure 4.4). The stretchable materials become functional if they are above a particular threshold $[94,104]$. The presence of the functional material in the elastic binder can limit the stretchability of the devices due to induced rigidity. Also, the conductivity may 
not completely recover after strain release, which is unfavorable for many applications [105].

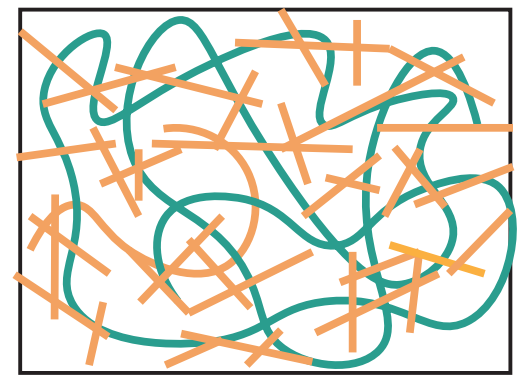

\section{Stretchable materials}

Functional networks

Figure 4.4. An illustration of a percolation network with a stretchable material.

\subsubsection{Buckled structures}

The strain on the conducting materials can be minimized by the use of buckled structures (Figure 4.5). Unfolding this structure can give a stable resistance throughout the stretching cycles. The conductivity can be kept stable with large strain through adjusting the pre-strain magnitude. Though it is proven as an efficient mechanism to reduce the negative influence on the functional material, the presence of the buckled structures increases roughness and reduce the transparency of the conductors which can limit the applications in transparent electrodes [106,107].

\section{Buckled film}

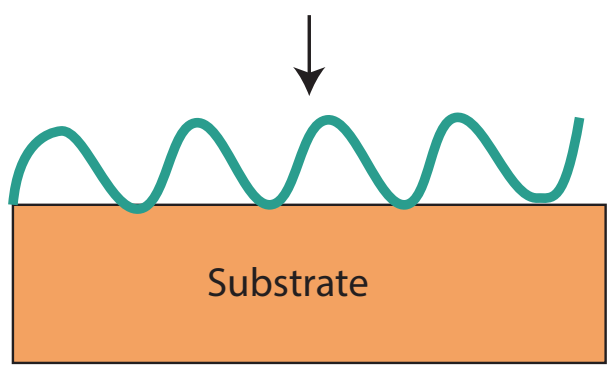

Figure 4.5. Illustration of a buckled film on a substrate.

\subsubsection{Serpentine structures}

The serpentine like structures helps the functional materials to withstand large strain and maintain a stable performance (Figure 4.6). This structure helps in precise control 
of the electromechanical performance under stretching cycles. The major challenge in these structures is the complicated fabrication [108].

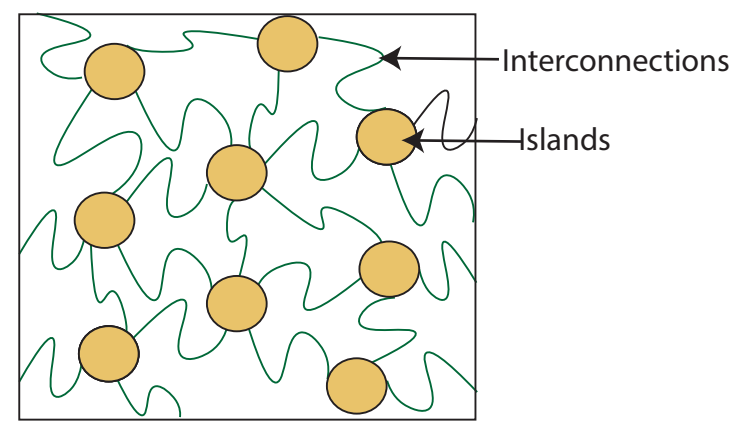

Figure 4.6. Illustration of serpentine structures.

\subsubsection{Helix structure}

Larger 3D helix structures can provide more space for deformation to withstand higher strains (fig 4.7). There has been recent works combining stretchable fibers with helix structures, which are good at withstanding larger number of stretches cycles. The improvement in the performance is mainly due to the elastic buffer connection of the helix structures, this structure alleviates the actual strain by revocable structural adaptation [109].

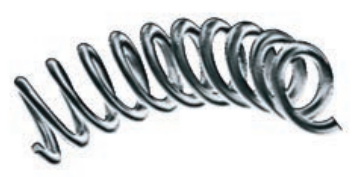

Figure 4.7. $3 \mathrm{D}$ helical stretchable structure. 
Part II

Experimental

techniques and results 



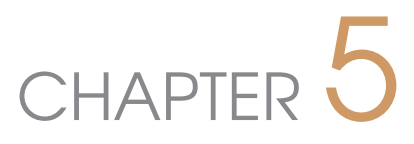

\section{Materials and}

\section{fabrication methods}

This chapter will introduce various kinds of materials used in the thesis and development of devices using various fabrication techniques, specific to development in the area of ion pumps and ion exchange membranes (IEMs). Methods for extracting data from the devices that we fabricated using the various characterization mechanisms will be covered in Chapter 7 .

\subsection{Materials}

\subsubsection{Substrates}

Depending on the kind of application we used different kind of substrates like glass, polyethylene terephtalate (PET), polyimide (PI), and polydimethylsiloxane (PDMS).

\subsubsection{Polyethylene terpthalate (PET)}

PET is the most common thermoplastic polymer of the<smiles>CCCOC(=O)c1ccc(C(=O)OCCO)cc1</smiles>

Figure 5.1. PET polyester family which is used extensively in fibers for clothing, containers, and thermoforming for manufacturing, and as a flexible substrate for various organicinorganic functional electronics applications [110, 111]. We used PET for the purpose of screen printing of functional inks on it (Paper 4), typically due to its flexibility and its excellent gas (and fair moisture) barrier properties. It also has excellent optical performance (high light transmittance, high smoothness, and low halo), and outstanding printability due to its surface energy [112]. All the films that we used were pre-conditioned in the oven at $130{ }^{\circ} \mathrm{C}$ for $1 \mathrm{~h}$ to avoid shrinking or other deformations during further processing. The PETs that we used in this thesis were all commercially available and had an extra coating to increase the surface energy of the PET to match the screen-printing requirements. 


\subsubsection{Polyimide (PI)}

$\mathrm{PI}$ is a polymer of imide monomers belonging to the class of high-performance plastics. It is used in various applications due to its excellent heat resistant properties. Polyimides exhibit an unusual combination of thermal stability $\left(>500{ }^{\circ} \mathrm{C}\right)$, mechanical toughness, and chemical resistance. They also have excellent dielectric properties $[113,114]$. Typical polyimide parts are not affected by commonly used solvents and oils - including hydrocarbons, esters, ethers, alcohols and freons [114]. The PI used in the development of in vitro ion pumps (Paper 3) was utilized in its solution phase, and was chosen for ease of integration with microfabrication protocols as well as<smiles>[R]C(=O)N([R2])C(=O)CC</smiles>

Figure 5.2. PI. due to its chemical inertness and biocompatibility. These PIs were cured after spin coating to achieve the desired thickness.

\subsubsection{Polydimethylsiloxane (PDMS).}

PDMS was chosen as the substrate for testing the stretchable membrane (Paper 2) and it is a mineral organic polymer of the siloxane family which is frequently used in microfluidic prototyping. PDMS is transparent, nontoxic, inert, and is has excellent visco-elastic properties $[115,116]$. PDMS has been well studied in stretchable electronics applications because of its tunable stretchable properties [117]. All the studies on stretchable membranes in this thesis were performed after oxygen plasma treatment of the PDMS. The oxygen plasma treatment

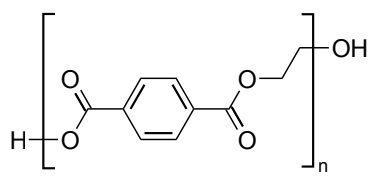

Figure 5.3. PDMS introduces polar functional groups $(\mathrm{SiOH})$ which change the intrinsic PDMS surface properties from hydrophobic to hydrophilic, facilitating further layer processing on the PDMS.

\subsubsection{Electrodes}

Different kinds of electrodes were used for devices fabricated. These include $\mathrm{Ag} / \mathrm{AgCl}$, PEDOT:PSS, gold, carbon paste, and silver. The electrodes for ion pumps need to be capable of converting electronic to ionic current and the device function thereby relies strongly on the electrodes' redox capacity: the measure of the materials' available sites for oxidation at anodic potentials or reduction at cathodic potentials [42]. For screen printed ion pump devices, silver was used as a contact electrode to the source meter, carbon is introduced between the silver and PEDOT:PSS to act as a buffer layer reducing the possibility of electrochemically released silver ions penetrating the PEDOT:PSS system. Here PEDOT:PSS acts as the redox active electrode. For 
stretchable OEIPs the electrode material used was $\mathrm{Ag} / \mathrm{AgCl}$, the electric current that reaches the $\mathrm{Ag} / \mathrm{AgCl}$ through the connection electrode is converted to ionic current by redox reaction, the ionic current then goes into the ion selective channels. For ion separation devices, gold electrodes were used for impedance measurements and $\mathrm{Ag} / \mathrm{AgCl}$ for the source and target electrodes. For in vitro ion pumps we used PEDOT:PSS coated PET sheets as the source and target electrodes for the ease of integration to the set-up.

\subsubsection{Ion exchange membranes (IEMs)}

In this thesis, over oxidized PEDOT:PSS, anionic hyperbranched polyglycerol (AHPG), and PSS-co-MA were used as ion selective membranes.

\subsubsection{PEDOT:PSS}

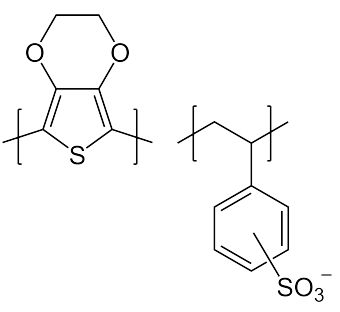

Figure 5.4. PEDOT:PSS.

Poly(3,4-ethylenedioxythiophene)-poly (styrene sulfonate) (PEDOT:PSS) is an organic semiconductor prepared by doping p-type conjugated polymer PEDOT with the polyanion PSS. PEDOT:PSS being a conjugated polymer blended with a polyelectrolyte has the versatile property of mixed ionic and electronic conductivity. PEDOT:PSS is solution processable and hence it is compatible with various coating and printing processes. It is an attractive candidate for stretchable and flexible thin film devices due to its conductive and electrochromic properties $[118,119]$. For the ion pumps described in Paper 4, the PEDOT:PSS is overoxidized by using sodium hypochlorite solution. In PEDOT:PSS, PEDOT offers the electric conductivity while PSS offers the ionic conductivity. The over oxidation breaks the $\pi$-conjugation and hence disables the electronic conducting pathways in the PEDOT while leaving the PSS phase undamaged. Thus the membrane becomes cation selective.

\subsubsection{Hyperbranched polyglycerol (HPG)}

Hyperbranched polymers consist of a category of polymers with an intrinsic structural arrangement based on a branching covalently bonded network. The HPGs used in this thesis (Figure 5.5) are materials synthesized specifically to be used as an ion selective membranes. These materials have excellent properties of forming stable electrophoretic channels with a high concentration of fixed charges $[120,121]$. HPGs can be made polyanionic or polycationic by functionalizing with negatively charged sulfonate group or positively charged trimethylphosphonium groups, depending on the requirements of charge transport. Furthermore, HPGs are capable of transporting 
larger or rigid aromatic ions by tuning the void fraction distribution and effective porosity of the bulk which is not possible with other linear polymers like PSS or PSSco-MA. For the in vitro (Paper 3 ) and stretchable (Paper 2) devices in this thesis, we used anionic HPGs as the channel material.

\subsubsection{PSS-CO-MA}

Poly (4-styrenesulfonic acid-co-maleic acid) (PSS-co-MA, Figure 5.5) mixed with polyethylene glycol (PEG), where the maleic acid forms ester bonds with - OH groups from PEG, forms an effective cation exchange membrane for iontronics [122]. After cross linking, it is available for further thin-film processing steps. PSS-co-MA/PEG is processed from solution by spin coating and hence it is well suited for photolithography patterning steps that follow. We used PSS-co-MA as an ion exchange membrane for ion separation devices (Paper 2).
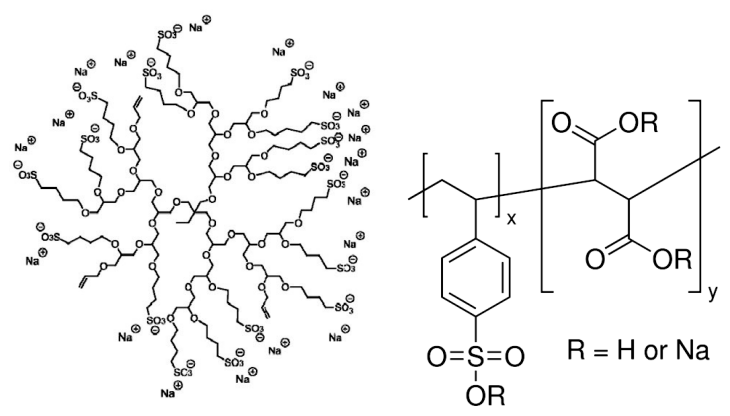

Figure 5.5. HPG (left) and PSS-co-MA (right).

\subsection{Fabrication methods}

\subsubsection{Screen printing}

Screen printing has been widely used in the field of printed electronics $[123,124]$ due to the technology's capability for fine adjustments of the film thickness and surface properties. It has been used for printing of electronic circuits since the 1940s [125]. In this thesis, screen printing is demonstrated as an efficient tool for printing of electronics and iontronic component for bioelectronic devices. Screen printing is the technique of transferring ink to a substrate through a mesh where select regions are made permeable to the ink enabling patterns in the final film (Figure 5.6). In practice, a squeegee is moved across the screen with ink which allows the screen to touch the substrate momentarily along a line of contact. This results in the transfer of ink 
through the screen to the substrate. The ink eventually dries on the substrate and forms the desired patterns. Screen printing thus essentially requires [126]: 1) a screen with desired patterns, 2) a squeegee, and 3) a substrate.

a

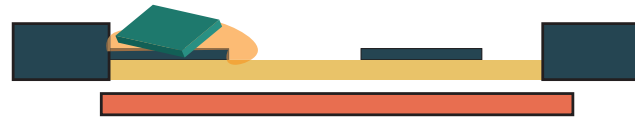

b

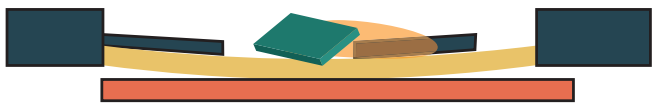

$\mathrm{C}$

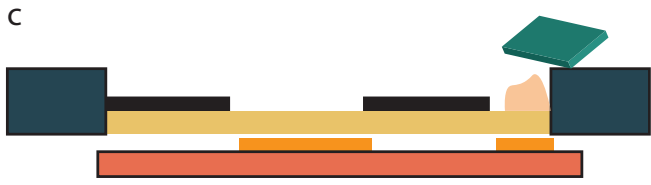

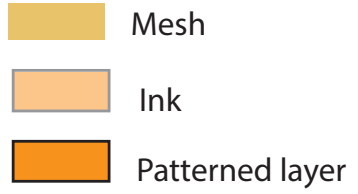

Squeege

Substrate

Figure 5.6. The screen printing process. a, Desired ink is applied on the screen. Required pressure and speed for printing are set using the control software. $\mathbf{b}$, Squeegee drags the ink across the whole screen covering the stencil. c, The screen revert back to the original length, the ink dries on the substrate to form the desired shape.

\subsubsection{Screen}

The screen consists of a screen-printing mesh and the frame that it is attached to. A screen frame upholds the structure of the screen. The frame should be able to hold the mesh without deforming itself and maintain the surface tension of the mesh throughout the printing process. For printed electronics applications, a metal or metal alloy mesh is usually used. The mesh holds the ink and controls the amount of ink that is transferred to the substrate. It should be flexible and resilient so that it can adapt to its original shape after the squeegee forces it to the substrate (Figure 5.6b). The choice of mesh influences the quality of print. A conventional mesh can be of stainless steel, nylon, or polyester. The material selection is based on the accuracy and the thickness of the required print, as well as on the type of ink (e.g., water based, solvent based, etc.). Other parameters that influence the printing are thread density and thread diameter of the mesh. 
The mesh diameter should be at least 3 times the particle size for the printing ink. Thinner lines can be printed with finer thread diameter. Thread density (or threads per centimeter) determines the amount of ink that is transferred to the substrate. Lower threads per centimeter results in more quantity ink transferred to the substrate and hence lower resolution. The mesh can also be mounted at different angles and, e.g., for printing circuits they are usually mounted at $45^{\circ}$ relative to the screen frame to share load over filaments and to prevent tension loosening in the mesh. The patterns created on the mesh are fabricated using photo-sensitive emulsions. The thickness of the emulsion should be chosen to form good printing, and adjusting the emulsion thickness will also help in tuning the print thickness.

\subsubsection{Squeegee}

The selection of the squeegee (or blade) is another important screen printing parameter that needs to be considered for print quality. Squeegee hardness and shape are the crucial parameters. The squeegee material should be flexible and resilient for a good quality screen printing. Another important parameter of the squeegee is that it should be chemically resistant to the ink being used. The most commonly used squeegee material is polyurethane and it can be selected with different hardness depending upon the screen and the ink. Selecting the proper degree at which the squeegee is applied over the mesh determines the effective pressure and thereby affects the print quality. In addition to the above, process parameters like squeegee speed, separation between the screen and the substrate (gap or "snap off"), applied squeegee pressure, and the rheological properties of the applied ink are parameters that can influence the print quality.

\subsubsection{Inkjet printing}

Inkjet printing is a material conserving non-contact technique for depositing liquid phase materials directly to a substrate. The material or ink that needs to be deposited is either dissolved or dispersed in a solvent. A specific quantity of materials that need to be deposited are ejected from the chamber. The drop falls under the influence of gravity and air resistance to reach the substrate. The drop then spreads on the substrate based on the momentum acquired during the fall, the surface tension of the drop, and the surface properties of the substrate. The drops then dry after solvent evaporation. The drop spreading and printed shape is also influenced by various parameters like viscosity of the liquid and print height [127]. A basic mechanism of ink deposition and drying using inkjet printing is illustrated in Figure 5.7 [128].

Inkjet printing technology can be classified mainly into two types: continuous mode inkjet (CIJ) and drop-on-demand (DoD). CIJ is used very rarely in the field of printed electronics. This technique creates a constant stream of small droplets of ink. These inks are charged and hence the droplets can be deflected by an electric field. The charged inks are deflected and deposited on the substrate to form the desired ink 
shape, while the uncharged ink goes to the feedback system. As the name suggest the, DoD method uses ink based on the requirements by the digital image. There are mainly two types of DoD systems, based on the print head they use: thermal and piezoelectric. Thermal DoD works using local heating and then evaporation of the liquid, the material or the ink that is used for deposition can either be in liquid or solid state.

In this thesis we only use DoD with piezoelectric print heads. Here, ink is ejected by sudden volume change initiated by piezoelectric action. The chamber containing the ink changes its shape after the applied voltage. This sudden shift in the volume as a result of the piezoelectric effect causes a shockwave in the liquid and ejects a drop from the nozzle. The piezoelectric mechanism of the inkjet printer is depicted in Figure 5.8.

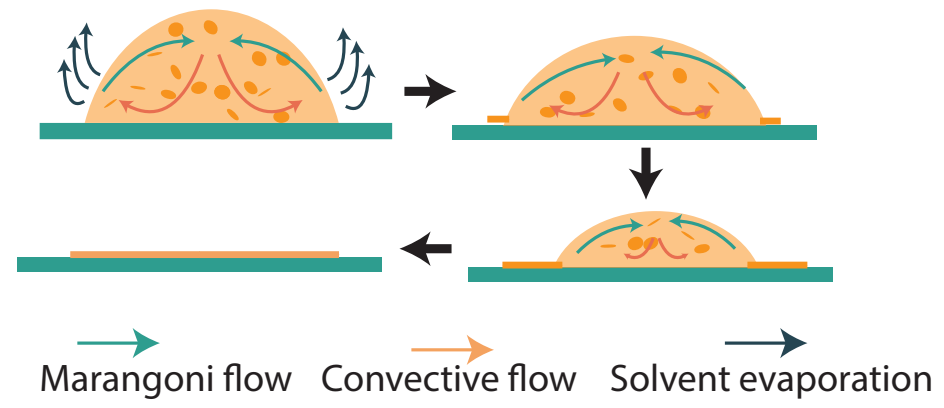

Figure 5.7. Possible drop drying mechanism after inkjet printing.

The thickness of the deposited ink $\left(T_{d}\right)$ is determined by the droplet volume $\left(V_{d}\right)$, concentration of the material in the ink $\left(C_{m}\right)$, and the number of drops deposited per unit area $\left(N_{d}\right)$ according to:

$$
T_{d}=V_{d} \times C_{m} \times N_{d}
$$

The main ink parameters that influence inkjet printing are viscosity, surface tension, particle content, polymer chain length, and ink evaporation rate [129]. It is not just the ink that determines the quality of the print; substrate properties like surface energy and surface roughness also influence inkjet printing to a large extent. Printer parameters also impact the print quality: drop volume, drop spacing (the centerto-center distance between two adjacent drops, directly related to print resolution), print head height (influences the momentum attained for the drop before it reaches the substrate), jetting frequency, firing voltage (voltage applied to the piezoelectric crystal). Higher firing voltage results in larger volume change, leading to quicker and larger drops with elongated tails, while lower firing voltage gives slower and smaller droplets with comparatively shorter tails (depending on the ink being used). 


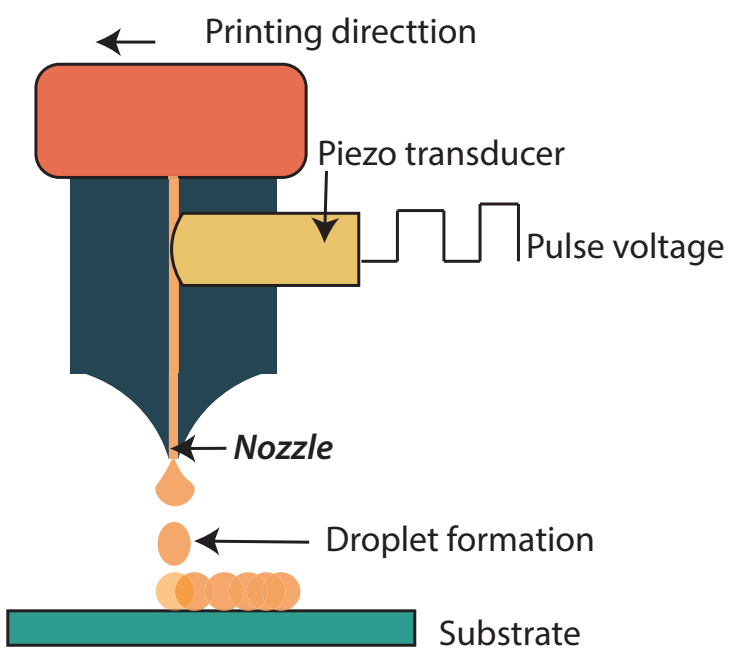

Figure 5.8. Basic mechanism of drop on demand piezo electric inkjet printing technique.

\subsubsection{Photolithography}

Photolithography, also called optical or UV-lithography, is a process widely used in microfabrication to pattern thin films. It transfers light through a geometrically patterned photomask to a photosensitive photoresist (chemical layer). A series of chemical treatments that follows after exposure either etch the exposed pattern or help in deposition of new layers or materials underneath the photo resist. Photolithography can be used for fabrication of highly complex integrated circuits [130]. A typical photolithography process workflow (Figure 5.9) could be outlined as follows: 1. cleaning, 2. photoresist spin coating, 3. exposure, 4. developing, 5. etching, 6. stripping. Organic and inorganic contaminants are initially removed from the substrate using wet chemical processes. In order to drive away the moisture content the substrate is usually heated. The photoresist is then applied by spin-coating technique (lamination or spray coating can also be used), where the photoresist is quickly ejected from the edge of the substrate and the bottom layer creeps radially along the wafer forming a uniform thin layer of photoresist. The viscosity and the spin-coating speed determines the final thickness of the photoresist deposited. For very small and dense structures less than $125 \mathrm{~nm}$, film thickness less than $500 \mathrm{~nm}$ is preferred. The spin coated photoresist is then post-baked to evaporate the excess solvent.

The spin coated photoresist is then exposed to light, causing chemical reactions in the photoresist which can then later be dissolved away with a special solution called developer. There are two kinds of photoresist: negative and positive. Negative photoresist becomes insoluble to the developer when exposed to the appropriate light, while the positive photoresist becomes soluble to the developer when exposed to light. 


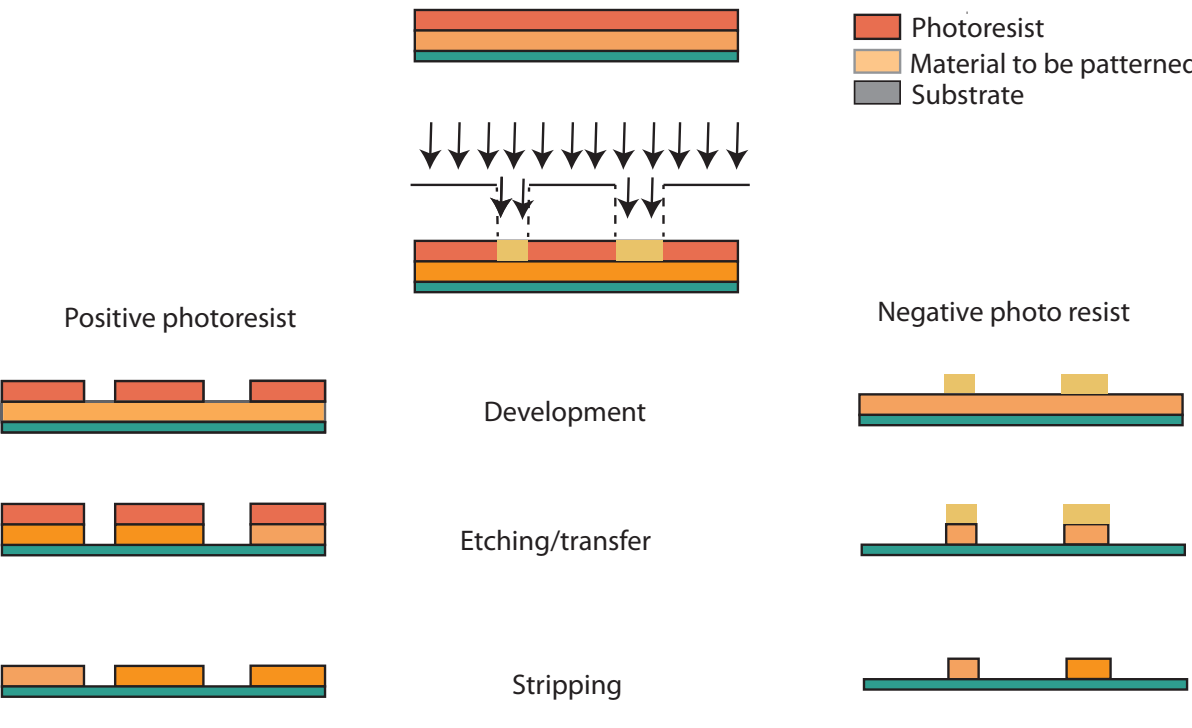

Figure 5.9. A typical photolithography process workflow. The arrows indicate UV/light exposure.

Depending upon the photoresist the mask films can be created to make patterns. The mask films are loaded in a mask aligner which helps in precision alignment with lower layers. The mask used can be made on plastic or glass depending on the requirement and resolution required for the pattern. Higher resolution can be achieved with chromium on glass (features $\geq 3 \mu \mathrm{m}$ ) or quartz (features $<3 \mu \mathrm{m}$ ). For less precision and lower resolution requirements, plastic masks can be used (features $\geq 10 \mu \mathrm{m}$ ). A post exposure bake (PEB) step is also introduced after the light exposure to avoid standing wave phenomena caused by interference patterns of the incident light. Developer solution is selected based on the chemistry of the photoresist used. There are mainly two kinds of etching depending on requirements: wet and dry etching. In wet etching, the sample surface is immersed in a solution which can chemically react with the substrate or the material to be patterned but remains chemically inert to the photoresist. In dry etching, the sample is bombarded by high energy ions in a vacuum environment. Elastic collision between ions and the surface atoms can cause many of the atoms to be removed from the surface. After the etching process, if the photoresist is no longer needed it is removed; this process is called stripping. Depending on the photo resist different types of resist stripper materials can be used. For all the devices described in this work, acetone was used as the resist stripper. 


\subsubsection{Physical vapor deposition (PVD)}

Physical vapor deposition (PVD) techniques are also used in conjunction with photolithography to deposit, e.g. thin metal films like gold $(\mathrm{Au})$, aluminium (Al), chromium $(\mathrm{Cr})$, or titanium $(\mathrm{Ti})$ in this work [130]. A PVD is a vaporization coating technique that involves transfer of material at the atomic level. PVD (Figure 5.10) can be outlined as follows: 1 . the material that needs to be deposited is converted to gaseous phase by high temperature vacuum or plasma; 2 . the material, in gaseous phase, is then transferred from the source to the substrate which is in the region of low pressure; 3 . the vapor will then undergo condensation on the substrate resulting in the formation of a thin film.

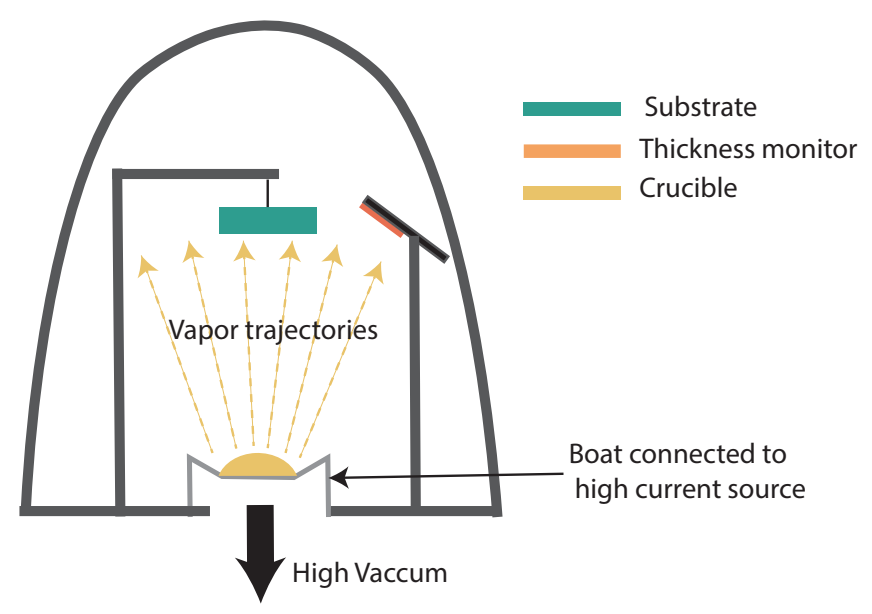

Figure 5.10. Physical vapor deposition (thermal evaporation).

PVD is used to deposit thin films of thickness ranging from just a few nanometers to several micrometers. PVD describes a wide range of deposition techniques that can generally be divided into evaporation and sputtering techniques. Evaporation mainly refers to deposition of materials with use of thermal means, while sputtering means deposition of material as a result of bombarding of high energy ions with the materials that need to be deposited. We primarily used thermal evaporation techniques for thin film deposition. With this process (Figure 5.10) the material to be deposited is converted from solid to vapor phase by application of high temperature (at low pressure). The source material typically in solid form is kept in a boat or a metal carrier, which is resistively heated to high temperature typically by the application of direct current. At the high vacuum created in the evaporation chamber, the material to be deposited converts to vapor phase, which then reaches the substrate and condenses there to form thin layer of material. This is usually used with materials with low melting point. 


\section{CHAPTER 6 \\ Devices developed in the thesis}

Four primary devices have been developed in thesis. These devices were fabricated using fabrication techniques discussed in the previous chapters. Fabrication schemes adapted for the device fabrication and the device architecture are explained through the schematics below.

\subsection{Iontronic separation}

a

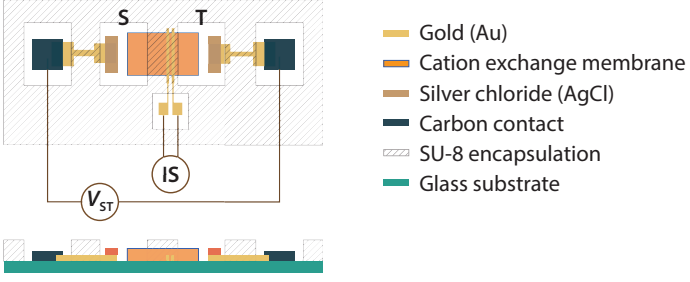

b

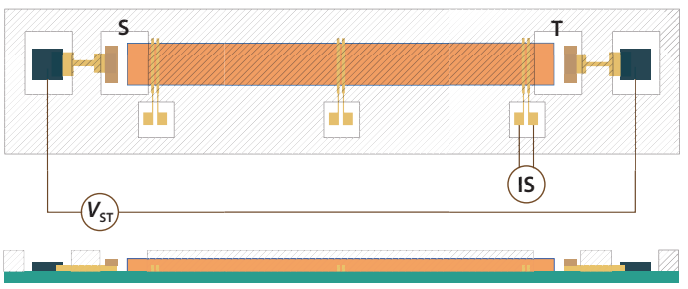

Figure 6.1. Top and side view of the iontronic chromatography devices fabricated in Paper 1. a, Devices used for single ion detection. $\mathbf{b}$, Devices used for multiple ion separation. $V_{S T}$ is the applied voltage between source and target, IS is the impedance spectroscopy measurement. Adapted from Paper 1.

Separation devices used in Paper 1 were fabricated serially in the order glass, 
electrodes, IEM, encapsulation. Au electrodes were initially patterned on glass substrates after thermal evaporation of $\mathrm{Cr} / \mathrm{Au}$. The patterned Au was used for applying voltage between source (anode) marked as $\mathrm{S}$ and target marked as $\mathrm{T}$ (cathode) in Figure 6.1. The electrode pair marked IS measured the impedance spectroscopy. Poly(4-styrenesulfonic acid-co-maleic acid) (PSS-co-MA) was used as the cation selective membrane (CEM). The preliminary study was executed with the device in Figure 6.1a with short CEM channels (5 $\mathrm{mm}$ long and $3 \mathrm{~mm}$ wide) to analyze the relationship between IS frequency and impedance with respect to different ions being transported. The longer-channel device (Figure 6.1b, $30 \mathrm{~mm}$ long CEM) was used for multiple ion detection. The sample solution or the analyte that needed to be separated was kept in the source reservoir and the target reservoir (T) was always $100 \mathrm{mM} \mathrm{KCl}(\mathrm{aq})$ in all experiments. Sample solution at $\mathrm{S}$ was switched to different mixtures while the voltage $V_{S T}$ was applied across $\mathrm{S}$ and $\mathrm{T}$, and the IS electrodes monitored the impedance at a constant frequency while ions were transported. Figure 6.2 shows the step-by-step fabrication protocol for the chromatographic devices.

a)

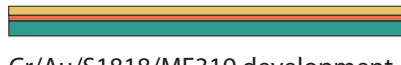

$\mathrm{Cr} / \mathrm{Au} / \mathrm{S} 1818 / \mathrm{MF} 319$ development

b)

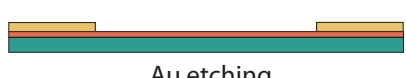

Au etching

c)

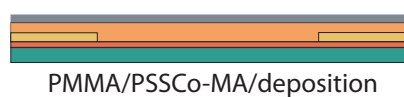

d)

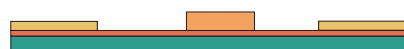

PSS-co-MA channel pattern /O2/CF4/ RIE

e)

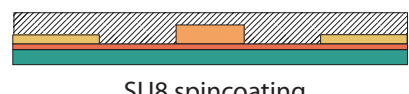

SU8 spincoating

f)

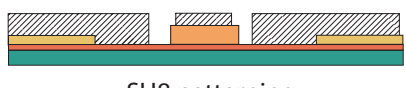

SU8 patterning

Figure 6.2. Iontronic chromatagraphy step-by-step microfabrication. a, $\mathrm{Cr} / \mathrm{Al}$ deposition by thermal evaporation followed by photopatterning using MF319 and S1818. b, Wet etching of $\mathrm{Al}$ using freshly prepared aluminium etchant. c, PSS-co-MA followed by PMMA deposition. d, Reactive ion etching to realize PSS-co-MA channels. e, SU-8 (encapsulation) deposition by spin-coating. f, SU-8 patterning by photo lithography. Adapted from Paper 1 . 


\subsection{Stretchable OEIP}

The stretchable OEIPs used in Paper 2 are shown in Figure 6.3. The final device is realized by sequential deposition of polydimethylsiloxane (PDMS) (by spin coating), the developed stretchable membrane based on hyperbranched polyglycerol (by stencil patterning), and PDMS (by spin coating.) The S and T are source and target reservoirs, and the target solution was kept at $100 \mathrm{mM} \mathrm{KCl} \mathrm{(aq)} \mathrm{for} \mathrm{all} \mathrm{the} \mathrm{measurements.}$ The source was switched depending upon the requirements. The voltage was applied between $\mathrm{S}$ and $\mathrm{T}$ through $\mathrm{Ag} / \mathrm{AgCl}$ electrodes (Ag wires immersed in ferric chloride solution for $5 \mathrm{~min}$ ). The devices were then clamped on a linear motorized stage and stretched to different strain values (see Section 7.3) while the ion pump current was monitored keeping constant voltage between $\mathrm{S}$ and $\mathrm{T}$. The step-by-step fabrication protocol for the devices is shown in Figure 6.4.

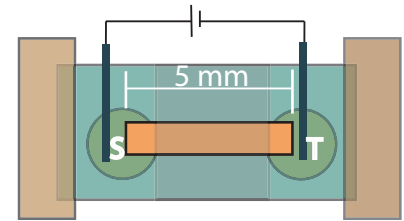

Before stretching

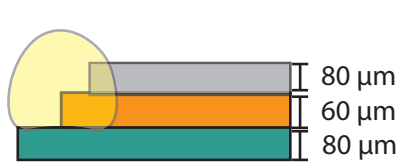

$\square$ Stretchable IEM

Substrate PDMS

PDMS Encapsulation

Silver/Silver chloride

Figure 6.3. Top and side view of stretchable OEIP used in Paper 2. The top view shows the device mounted on linear stretching setup with the $\mathrm{Ag} / \mathrm{AgCl}$ electrode connected to the external power source. Adapted from Paper 2.

a)

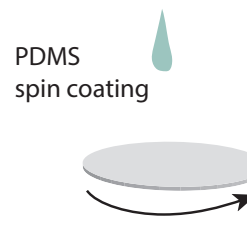

f)

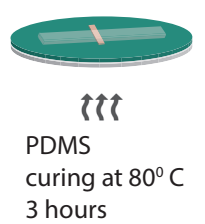

b)

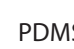

curing at $80^{\circ} \mathrm{C}$

3 hours

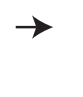

e)

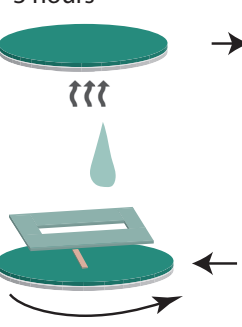

PDMS

Spin coating with Mask

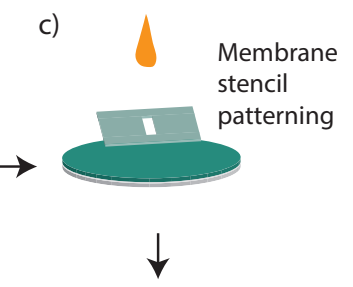

d)

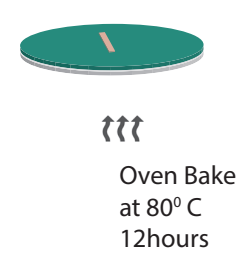

Figure 6.4. Stretchable OEIP fabrication. Adapted from Paper 2. 


\subsection{In vitro flexible ion pump}

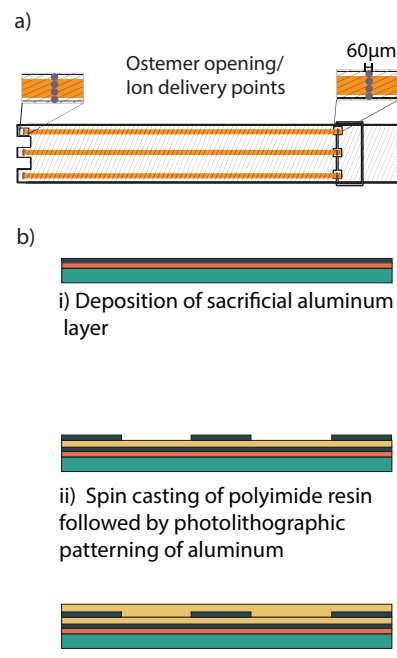

iii) Spincasting of polyimide

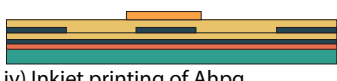

iv) Inkjet printing of Ahpg

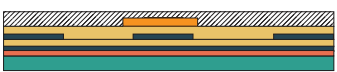

v) Wire bar coating of ostemer 221 encapsulant
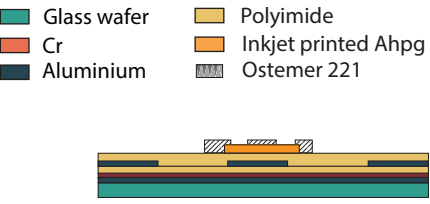

vi) Photo lithographic patterning of ostemer 221 encapsulant

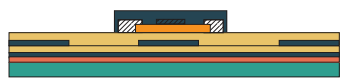

vii) Photolithographic patterning of aluminum protection layer

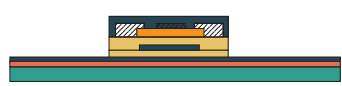

viii) Reactive ion etch cutout

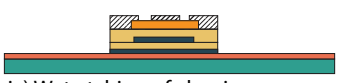

ix) Wet etching of aluminum

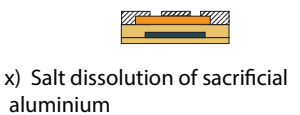

Figure 6.5. a, Top and side view of flexible OEIP for in vitro studies (Paper 3). Three ion channels patterned using inkjet printing have delivery outlets both on source and target sides. The zoomed-in image shows the delivery points. Each channel has 4 delivery points $(60 \mu \mathrm{m}$ diameter) created by photo-patterned Ostemer encapsulant. b, Fabrication steps for flexible ion pumps. i, Chromium (5 nm) and sacrificial aluminum $(50 \mathrm{~nm})$ deposition by thermal evaporation on glass wafer. ii, Spin-casting of polyimide followed by aluminum thermal evaporation $(50 \mathrm{~nm})$ and photopatterning for alignment. iii, Spin-casting of polyimide to cover the aluminum alignment layer. iv, Inkjet printing of anionic hpg (Ahpg) printing and UV crosslinking. v, Wire bar coating of Ostemer 221 encapsulant to fulfill thicker layer $(9 \mu \mathrm{m})$ to cover the bottom Ahpg. vi, One step UV-patterning of Ostemer 221 to realize the source and target outlets. vii, Aluminum deposition by thermal evaporation $(70 \mathrm{~nm})$ and photo patterning for protection layer to define device cut out. viii, Reactive ion etching to cut out the devices, the areas which are not protected by the aluminum are etched through. ix, Wet etching of aluminum removes all the aluminum protection. $\mathbf{x}$, Salt dissolution using $\mathrm{NaCl}$ strips off the device from the aluminum bottom layer. Adapted from Paper 3. 
The free standing fully flexible ion pump used in Paper 3 for in vitro applications is shown in Figure 6.5a. It was realized through a set of coating and deposition protocols including photo patterning, inkjet printing, wire bar coating, and spin casting. The elaborated fabrication protocol is illustrated in Figure 6.5 b. For these ion pumps, the delivery outlets are formed by patterned encapsulant (here, we used a proprietary material Ostemer). The characterization of these devices was done by sealing the fabricated ion pump in a heat shrinkable tube, with the delivery points outside the tube and source delivery outlets inside the tube. Bupivacaine was kept inside the shrink tube and delivered to either target dorsal root ganglion (DRG) cells in cell medium or $100 \mathrm{mM} \mathrm{KCl} \mathrm{(aq).} \mathrm{The} \mathrm{source} \mathrm{and} \mathrm{target} \mathrm{were} \mathrm{connected} \mathrm{to} \mathrm{a} \mathrm{source}$ meter through PEDOT electrodes (applying constant current). The DRG response was monitored through a confocal microscope (using $\mathrm{Ca}^{2+}$ imaging) and the voltage response was monitored with the source meter.

\subsection{Fully screen-printed ion pump}

The fully screen printed OEIPs used in Paper 4 are shown in Figure 6.6. They were fabricated on flexible PET substrates using only sequential printing of contact pads, ion selective membranes, organic electrodes, and dielectric materials as shown in Figure 6.6. As explained for the other devices, the $\mathrm{S}$ indicates source and $\mathrm{T}$ indicates target reservoirs. Constant voltage or current was applied between silver contacts using a source meter for characterization. Here, screen printed PSS (over-oxidized PEDOT:PSS) was used as a CEM and screen printed pDADMAC was used as an AEM. Devices were characterized electrically and delivery was chemically quantified using ELISA (for acetylcholine delivery) and commercial biosensors (for salicylic acid delivery). 
a)

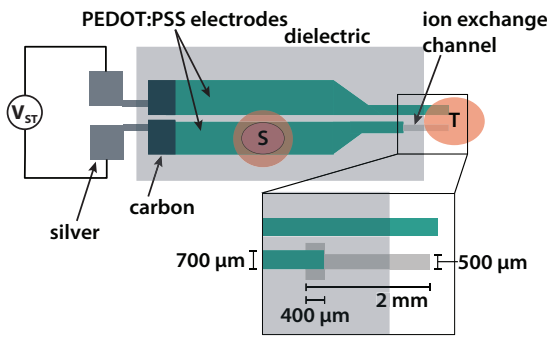

b)

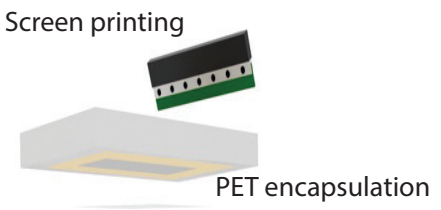

Adhesive

Target electrolyte

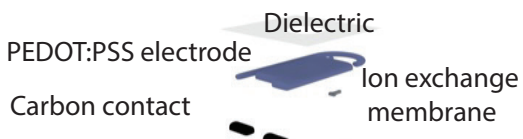

Silver electrode

PET substrate

Figure 6.6. a, Top view of the screen printed OEIP used in Paper 4. $V_{S T}$ is the applied voltage between source and target, $\mathrm{S}$ and $\mathrm{T}$ are the sealed source and target reservoirs. The zoomed in area shows specific dimension of the ion exchange membranes and electrodes.b, The screen printing process flow starts from the pre-treated PET substrate followed by sequential screen printing of silver and carbon contacts, ion exchange membrane (pDADMAC ${ }^{+}$or $\mathrm{PSS}^{-}$), PEDOT:PSS electrodes, and dielectric. Electrolytes deposited and UV cured to form gel and enclosed with adhesive layers and a PET lid. The final individual devices are obtained by high resolution laser cutting. Adapted from Paper 4. 


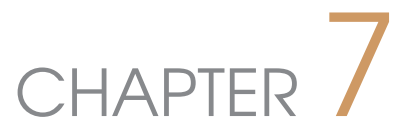

\section{Characterization}

\subsection{Electrical characterization}

\subsubsection{Voltage and current measurement}

The voltage and current measurement done in this thesis used a source meter in a 2-probe measurement set up. This setup could be used in constant current or constant voltage mode for OEIPs and for experiments on ion separation. The ionic transport properties were electrically analyzed by assuming the current output to be proportional to the number of ions transported. The input was controlled and monitored by a custom designed LabVIEW program. The voltage or current was applied between the source and target, the source electrode was either positively or negatively biased depending on if cations or anions were to be transported (which was also contingent on if the IEM was an AEM or CEM). As the ionic conductivity is very low, it was important to use sophisticated electrical characterization equipment like the source meter as its possible to measure ionic currents as low as $5 \mathrm{nA}$.

\subsubsection{Impedance spectroscopy (IS)}

IS measures impedance of a system in dependence of AC voltage at a range of frequencies. Electrochemical IS (EIS) is the response of an electrochemical system to an applied potential, where the frequency dependence of this response can reveal fundamental chemical processes [131]. Impedance is calculated from the ratio of frequency-dependent voltage to the frequency-dependent current. A measurement setup consisting of reference electrode, working electrode, and counter electrode is generally used for IS. The impedance value is represented as a complex number $\mathbf{Z}$, which is represented as a vector of length $\mathbf{Z}$ in the complex plane with angle $\phi$ relative to the real axis. $\mathbf{Z}$ can also be represented as the real part of the impedance, $\mathbf{Z}$ ', which is the resistance, and an imaginary part, Z". A known sinusoidal AC voltage is applied to the counter electrode through a system or electrolyte solution from the working electrode. Then EIS measures the current wave generated, resulting in quantitative measurements (Z, Z', Z' '), and $\phi$ are calculated and plotted against different frequency) and this enables the analysis and evaluation of small scale electrochemical process at the interface of the electrode as well as within the system. 


\subsection{Optical characterization}

\subsubsection{Surface and rheological studies}

Surface tension, viscosity and contact angle measurements are performed in this work for layer compatibility, adhesion between different materials, process compatibility, and ink characterization for different printing techniques. As discussed in Chapter 5, ink rheology is very much important for both screen and inkjet printing. For inkjet printing, viscosity helps an ink to be pulled into and pushed out of the nozzle. The ink viscosity should be in a range (for our studies, 6-14 cps) that supports good drop formation. The surface tension should be high enough to hold the ink without dripping and should not be too high to hinder the drop from splitting apart from the bulk (for studies in this work, 20-42 dynes/cm) [129]. For screen printing the viscosity affects the ease at which the ink passes through the mesh, surface tension is also important which determines the ink adhesion to the substrate and how much ink is held back in the screen. It is important to know the ink and substrate interaction when the ink reaches the substrate. This is achieved by measuring the liquid contact angle, $\theta$. If $90^{\circ}<\theta<150^{\circ}$ the substrate is considered to be hydrophobic, and if $\theta>150^{\circ}$ the substrate is super hydrophobic. $\theta<90^{\circ}$ indicates hydrophilicity of the substrate [128]. Contact angle study gives information about the nature of the substrates. For most of the printing requirements, a hydrophylic substrate is preferred for ink spreading and layer formation. Measurements on contact angle and surface tension were performed with a contact angle meter (goniometer). Viscosity and viscoelastic measurements were done using a rheometer. The thickness of the deposited materiel and their morphology was studied using surface and optical profilometers and optical microscopes.

\subsubsection{Fluorescence microscopy}

Fluorescence microscopy was used in this thesis for in vitro studies after delivering nerve blocking agent (bupivacaine) to mouse DRG cells (Paper 3). As the name says, fluorescence microscopy is an optical microscopy method which uses fluorescence to study the properties of organic and inorganic substances [132]. Basic fluorescence microscopy can be explained based on the Figure 7.1. The sample containing fluorophores absorbs a light of specific wavelength when illuminated and this causes the fluorophores to emit light of different wavelength. The emitted light is differentiated from other light using an emission filter. The emission filter and dichroic mirror are chosen to match the fluorophores used for the samples. In this way a single fluorophore species is recorded at a time. Multicolor images with multiple fluorophores can be made by combining several images. The light source can be, e.g., a xenon arc lamp, mercury lamp, laser, high power LEDs, etc. For our measurements, we used a confocal microscopy system with a laser source for fluorescence measurements. In conventional fluorescence systems all part of the sample to be analyzed are illuminated 


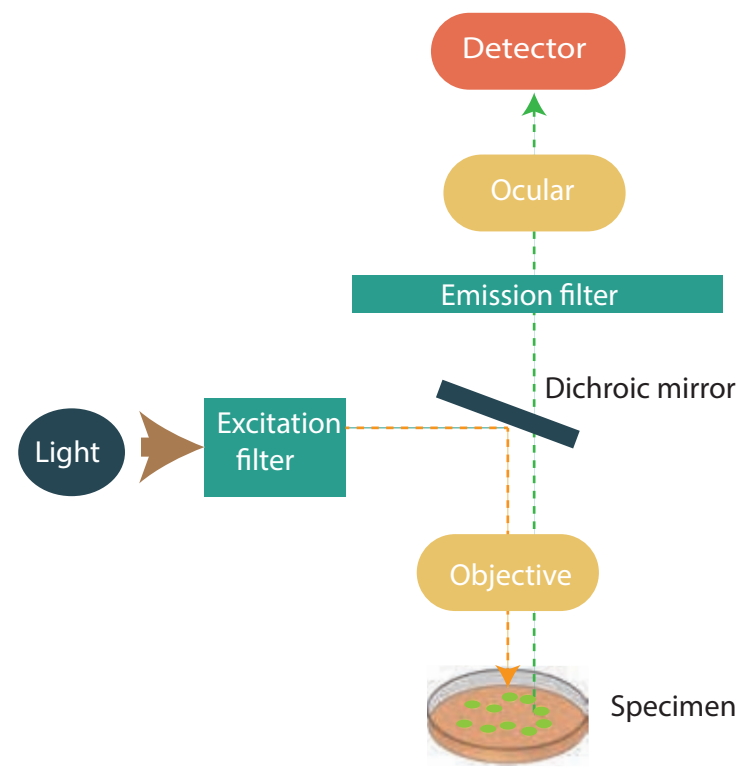

Figure 7.1. Schematics of a fluorescence microscope.

by a light source and the entire excited region including the unfocused background is recorded. In contrast, the confocal microscopy eliminates out of focus background and narrows the depth of field to a single plane.

\subsection{Linear stretching system}

A linear motorized stretching system was used for characterization of the stretchable ion exchange membrane we developed in Paper 2. A basic schematic of the system is shown in Figure 7.2. The device to be characterized was mounted on the system, consisting of a fixed clamp and a moving clamp with a motor controlled by a computer program. A standard experiment would stretch the membrane to different strain values and release it to $0 \%$ strain and could be repeated several times to understand the stretchable properties of the membrane. The speed at which the membrane was stretched to different strain value could be varied depending upon the experimental requirements. The ionic conductivity was measured simultaneously using a source meter at constant applied voltage. The setup also had a force meter to measure the stress to the applied strain. The Young's modulus of the membrane was calculated from these stress and strain measurement values. 


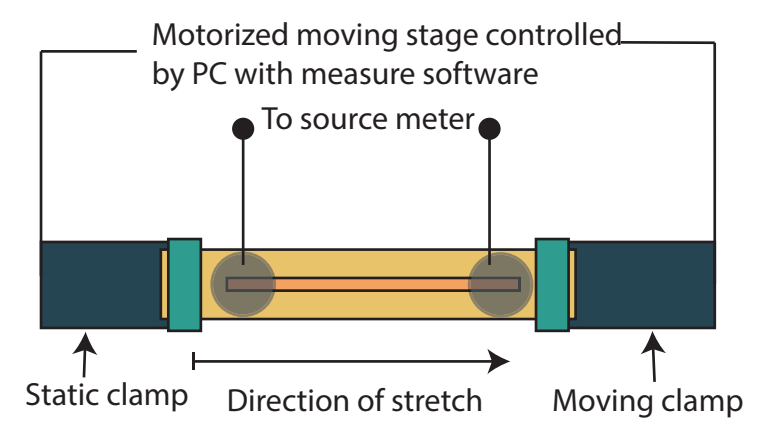

Electrolyte $\square$ Ion channel

Figure 7.2. Illustration of a linear stretching set up. 


\section{CHAPTER 8 \\ Concluding remarks}

This thesis is an abstract of my past four years of work on the remarkable field of iontronics. Here, I was able to step into the field of iontronic delivery devices and give a different methodology on perceiving and studying these devices, not only in the perspective of functionality but also in manufacturing. There has been vast research on using iontronic devices for bio-relevant transport and delivery, but less attention has been given to manufacturing these devices and expanding functionality other than drug delivery.

\subsection{Conclusion}

In this work we were able to offer an addition to the iontronics family: the first analytical detection technique for fluid-less fully-electronic ion detection. Paper 1 discusses these devices and techniques which we named iontronic separation. With the iontronic platform we proved the individual detection of ions with impedance as a signature. More particularly we also demonstrated that ionic species can be distinguished based on their diffusion coefficient. Multiple ionic detection for organic molecular ions $(\mathrm{ACh})$ and for ions that are relevant for drinking water $\left(\mathrm{K}^{+}, \mathrm{Ca}^{2+}\right.$, and $\mathrm{Na}^{+}$) was also demonstrated in this work. The device operates at a much lower voltage $(20 \mathrm{~V})$ compared to conventional separation techniques like capillary electrophoresis $(\sim 10 \mathrm{kV})$ and with quicker detection speed $(1 \mathrm{~s}-1 \mathrm{~min})$. These studies imply that we can certainly get peak separation and that it is possible to detect multiple alkali ions mixed at different ratios. These proof-of-concept studies used CEMs for detection of cations which could be further expanded with AEMs. Moving forward with iontronic devices, we tried to develop a delivery device with a form factor ideal for biological applications. There have been extensive studies conducted on the development of stretchable electronic materials, but less focus has been on stretchable ion exchange membranes - although such materials could find enormous applications in the field of organic bioelectronics and could be used in association with many stretchable electronics applications like stretchable displays and energy storage devices. Current ion pumps lack the conformability and stretchability to be used for implantable drug delivery application in, e.g., lungs, heart, muscle, soft or brain implants, joints. Due to the brittleness of the crosslinkers and the ionic materials that are currently used for ion selective membranes, they cannot easily be used in the above 
kinds of applications. Keeping this in mind, we present a stretchable IEM in Paper 2, which was developed with hyperbranched polyglycerol (HPG) as the base membrane material (a well-studied material for iontronics and transport of larger ions) and waterbased polyurethane (WPU) as a main component. The stretchable properties of the membrane were tested and established by fabricating an ion pump on a stretchable PDMS substrate. Devices were successfully demonstrated as a stretchable skin patch mounted on human thumb, with the capability of ionic delivery while being stretched. We progressed deeper into the fabrication of OEIPs using the tailor-made HPG IEMs developed within our lab for iontronics. We developed a fully flexible ion pump with inkjet printing (HPG-based inkjet ink) and micro-fabrication techniques particularly for in vitro studies. The ion pump was then tested for ion selective transport and demonstrated for high-precision in vitro delivery of bupivacaine to primary mouse dorsal root ganglion (DRG) cells. The architecture and design of the fabricated ion pump allowed local monitoring of cells' response to drug delivery. We were able to deliver bupivacaine vertically within $100 \mu \mathrm{m}$ of the cells. We also observed highly local response (approx. $120 \mu^{2}$ area) and that delivery charge as low as $30 \mu \mathrm{C}$ being suitable to create a cell response to bupivacaine delivery. Further increase in the charge delivered did not create significant reduction in the cell response and region that is away from the delivery point did not show considerable effect, validating the prospective of ion pump for high precision and fast delivery. After studying and developing flexible ion pumps for high precision delivery using micropatterning techniques, we addressed the need of developing a large-scale manufacturing technique. A novel approach on fabrication of the OEIP was adapted for large-area manufacturing using screen-printing technology. We demonstrated completely encapsulated flexible OEIPs fabricated through a screen-printing process favorable for large-scale manufacturing. This screen-printing design established a system with enclosure and gelling to alleviate malfunction by leakage, as well as a geometry allowing fast filling of electrolytes for swift testing. The fabrication process was effortlessly tunable by screen-printing of two different ion exchange membrane formulations and integration into the same design. These screen-printed OEIPs could thus be used to deliver either cations or anions, using a PSS CEM or pDADMAC AEM. The printed design gave a manufacturing yield $>90 \%$. These devices were successfully quantified for the transport of cationic neurotransmitter acetylcholine, and the anionic anti-inflammatory salicylic acid, demonstrating the potential for iontronics to be manufactured on flexible substrate at an industrial scale.

\subsection{Outlook}

Based on all the works that are presented in this thesis I believe this has opened the door for immense research and application possibilities. For the iontronic chromatography presented in Paper 1 as a new application for the OEIP family, with further improvement to the device geometry, measurement technique, and calibra- 
tion, I believe this technique could be used for detection of more than two species. For upscaling and effective applications, we need to overcome many designs and technical hurdles such as $(i)$ keeping the long ion exchange membrane hydrated to have a constant transport of ions, requiring better encapsulation or a hydration environment; (ii) an automated microfluidic system for ion exchange or ion switch for the analyte. Furthermore, the size of the ionic species that could be transported and separated by the ionic chromatography device in Paper 1 is limited by the IEM materials. This limitation could be solved by incorporation of the HPG-based IEMs used in Papers 2 and 3. Due to the straightforward fabrication techniques, simple geometry, and low operating voltage this developed detection technique could be adapted to portable applications like wastewater sampling, and ion detection in biological samples. The stretchable ion exchange membranes developed in Paper 2, on the other hand, with the established ion transport properties and softness of the IEMs discussed, could find exciting application in stretchable devices ranging from stretchable displays, to energy storage devices, biomedical implants, etc. The developed ion exchange membrane is based on labor-intensive manual fabrication like spin-coating and stencil patterning. These could be upscaled to larger-scale fabrication by developing a screen-printable formulation with the membranes. An approach in this direction will plausibly help in finding applications of these membranes in new areas. In the case of ink jet printable membrane for in vitro delivery (Paper 3) the ease of integration of several fabrication protocols and substrates can be used as a testing platform of new ion exchange membranes and architectures. The developed inkjet formulation can be adapted to any devices and designs. With the mechanical flexibility and biocompatibility of the demonstrated ion pump, and with the design freedom of the fabrication protocol developed, these ion pumps can be easily adapted for in vivo devices. The design and architecture demonstrated for the ion pump provide a platform for cell study and understanding high precision electrophoretic delivery of ions using ion pumps. In the final paper on large-area ion pump (Paper 4), I believe in addition to developing a robust fabrication protocol for mass manufacturing of iontronics, the bio-relevant ions delivered highlight potential applications in neuroscience, inflammatory disease, and epidermal wound healing. Combining the large area manufacturing capability on flexible substrates and epidermal healing effects of salicylic acid, these could find exciting applications in smart wound care applications. However, as most of the printed materials are commercially available and the biocompatibility of these materials are somewhat unknown, there is need for more study testing for biocompatibility before getting into in vivo/implant applications. This screen-printing protocol can be combined with other fabrication procedures that we have used for other devices in this thesis like inkjet printing and photo patterning for improved delivery efficiency, and the methods could even be used to find exciting in vivo applications due to the freedom of design and form factor they can achieve. 


\section{References}

[1] C. Nicolini, "From neural chip and engineered biomolecules to bioelectronic devices: An overview," Biosensors and Bioelectronics, vol. 10, pp. 105-127, jan 1995 .

[2] J. Rivnay, R. M. Owens, and G. G. Malliaras, "The rise of organic bioelectronics," jan 2014.

[3] P. Chen, X. Sun, and H. Peng, "Emerging Soft Bioelectronics," jul 2020.

[4] E. D. Głowacki, E. Stavrinidou, and D. Khodagholy, "Bioelectronics Research Reaches New Heights," mar 2020.

[5] F. Fu, J. Wang, H. Zeng, and J. Yu, "Functional Conductive Hydrogels for Bioelectronics," oct 2020.

[6] K. R. Harris, "Comment on "Ionic Conductivity, Diffusion Coefficients, and Degree of Dissociation in Lithium Electrolytes, Ionic Liquids, and Hydrogel Polyelectrolytes"," The Journal of Physical Chemistry B, vol. 122, pp. 1096410967, dec 2018.

[7] H. Kai, W. Suda, Y. Ogawa, K. Nagamine, and M. Nishizawa, "Intrinsically Stretchable Electrochromic Display by a Composite Film of Poly $(3,4-$ ethylenedioxythiophene) and Polyurethane," 2017.

[8] K. Feron, R. Lim, C. Sherwood, A. Keynes, A. Brichta, and P. C. Dastoor, "Organic bioelectronics: Materials and biocompatibility," aug 2018.

[9] S. Löffler, B. Libberton, and A. Richter-Dahlfors, "Organic bioelectronic tools for biomedical applications," nov 2015.

[10] F. Bischoff, "Organic Polymer Biocompatibility and Toxicology," Clinical Chemistry, vol. 18, no. 9, pp. 869-894, 1972.

[11] B. Ř́hová, "Biocompatibility of biomaterials: hemocompatibility, immunocompatiblity and biocompatibility of solid polymeric materials and soluble targetable polymeric carriers," Advanced Drug Delivery Reviews, vol. 21, no. 2, pp. 157-176, 1996. 
[12] M. Berggren and A. Richter-Dahlfors, "Organic bioelectronics," Advanced Materials, vol. 19, pp. 3201-3213, oct 2007 .

[13] D. T. Simon, E. W. H. Jager, and K. Tybrandt, "An organic electronic ion pump to regulate intracellular signaling at high spatiotemporal resolution," 2009.

[14] D. C. Martin, "Molecular design, synthesis, and characterization of conjugated polymers for interfacing electronic biomedical devices with living tissue," $M R S$ Communications, vol. 5, pp. 131-153, jun 2015.

[15] A. J. Teo, A. Mishra, I. Park, Y. J. Kim, W. T. Park, and Y. J. Yoon, "Polymeric Biomaterials for Medical Implants and Devices," apr 2016.

[16] A. Bashir, T. I. Awan, A. Tehseen, M. B. Tahir, and M. Ijaz, "Interfaces and surfaces," in Chemistry of Nanomaterials, pp. 51-87, Elsevier, jan 2020.

[17] S. Lee, D. Sasaki, D. Kim, M. Mori, T. Yokota, H. Lee, S. Park, K. Fukuda, M. Sekino, K. Matsuura, T. Shimizu, and T. Someya, "Ultrasoft electronics to monitor dynamically pulsing cardiomyocytes," Nature Nanotechnology, vol. 14, no. 2, pp. 156-160, 2018.

[18] W. S. Wong and A. Salleo, Flexible Electronics: Materials and Applications. 2009.

[19] A. Jonsson, Z. Song, D. Nilsson, B. A. Meyerson, D. T. Simon, B. Linderoth, and M. Berggren, "Therapy using implanted organic bioelectronics," Sci. Adv., 2015.

[20] Z. Zhu, R. Li, and T. Pan, "Imperceptible Epidermal-Iontronic Interface for Wearable Sensing," Advanced Materials, vol. 30, no. 6, p. 1705122, 2017.

[21] T. Arbring Sjöström, M. Berggren, E. O. Gabrielsson, P. Janson, D. J. Poxson, M. Seitanidou, and D. T. Simon, "A Decade of Iontronic Delivery Devices," Adv. Mater. Technol., vol. 3, p. 1700360, may 2018.

[22] Y. Chang, L. Wang, R. Li, Z. Zhang, Q. Wang, J. Yang, C. F. Guo, and T. Pan, "Interfacial Iontronic Sensing: First Decade of Interfacial Iontronic Sensing: From Droplet Sensors to Artificial Skins (Adv. Mater. 7/2021)," Advanced Materials, vol. 33, no. 7, p. 2170050, 2021.

[23] E. O. Gabrielsson and M. Berggren, "Polyphosphonium-based bipolar membranes for rectification of ionic currents," Biomicrofluidics, vol. 7, p. 064117, nov 2013.

[24] A. Jonsson, T. A. Sjöström, K. Tybrandt, M. Berggren, and D. T. Simon, "Chemical delivery array with millisecond neurotransmitter release," 
[25] K. Tybrandt, E. O. Gabrielsson, and M. Berggren, "Toward complementary ionic circuits: The npn ion bipolar junction transistor," J. Am. Chem. Soc., vol. 133, pp. 10141-10145, jan 2011.

[26] J.-H. Han, K. Kim, H. Kim, and T. Chung, "Ionic Circuits Based on Polyelectrolyte Diodes on a Microchip," Angew. Chemie Int. Ed., vol. 48, pp. 3830-3833, may 2009 .

[27] K. Tybrandt, K. C. Larsson, A. Richter-Dahlfors, and M. Berggren, "Ion bipolar junction transistors," Proc. Natl. Acad. Sci., vol. 107, pp. 9929-9932, jan 2010.

[28] G. Sun, S. Senapati, and H. C. Chang, "High-flux ionic diodes, ionic transistors and ionic amplifiers based on external ion concentration polarization by an ion exchange membrane: A new scalable ionic circuit platform," Lab Chip, vol. 16, no. 7, pp. 1171-1177, 2016.

[29] X. Strakosas, M. Seitanidou, K. Tybrandt, M. Berggren, and D. T. Simon, "An electronic proton-trapping ion pump for selective drug delivery," Science Advances, vol. 7, jan 2021.

[30] T. Sata, "Studies on anion exchange membranes having permselectivity for specific anions in electrodialysis - effect of hydrophilicity of anion exchange membranes on permselectivity of anions," Journal of Membrane Science, vol. 167, pp. 1-31, mar 2000.

[31] J. M. Leger, M. Berggren, and S. A. Carter, Iontronics: Ionic Carriers in Organic Electronic Materials and Devices. Boca: CRC Press, 2011.

[32] I. Uguz, C. M. Proctor, V. F. Curto, A. M. Pappa, M. J. Donahue, M. Ferro, R. M. Owens, D. Khodagholy, S. Inal, and G. G. Malliaras, "A Microfluidic Ion Pump for In Vivo Drug Delivery," Adv. Mater., vol. 29, no. 27, pp. 1-6, 2017.

[33] J. Isaksson, P. Kjäll, D. Nilsson, N. Robinson, M. Berggren, and A. RichterDahlfors, "Electronic control of Ca2+ signalling in neuronal cells using an organic electronic ion pump," Nat. Mater., vol. 6, pp. 673-679, sep 2007.

[34] A. Williamson, J. Rivnay, L. Kergoat, A. Jonsson, S. Inal, I. Uguz, M. Ferro, A. Ivanov, T. A. Sjöström, D. T. Simon, M. Berggren, G. G. Malliaras, and C. Bernard, "Controlling epileptiform activity with organic electronic ion pumps," Adv. Mater., vol. 27, no. 20, pp. 3138-3144, 2015.

[35] D. Cherian, A. Armgarth, V. Beni, U. Linderhed, K. Tybrandt, D. Nilsson, D. T. Simon, and M. Berggren, "Large-area printed organic electronic ion pumps," Flex. Print. Electron., vol. 4, p. 022001, apr 2019.

[36] T. Arbring Sjöström, A. Jonsson, E. Gabrielsson, L. Kergoat, K. Tybrandt, M. Berggren, and D. T. Simon, "Cross-Linked Polyelectrolyte for Improved Selectivity and Processability of Iontronic Systems," ACS Appl. Mater. Interfaces, vol. 9, pp. 30247-30252, sep 2017. 
[37] K. Tybrandt, K. C. Larsson, A. Richter-Dahlfors, and M. Berggren, "Ion bipolar junction transistors," PNAS, vol. 107, no. 22, pp. 9929-9932, 2010.

[38] J. Ran, L. Wu, Y. He, Z. Yang, Y. Wang, C. Jiang, L. Ge, E. Bakangura, and T. Xu, "Ion exchange membranes: New developments and applications," 2016.

[39] E. O. Gabrielsson, "Monopolar and Bipolar Membranes in Organic Bioelectronic Devices," 2014.

[40] J. Kamcev, D. R. Paul, G. S. Manning, and B. D. Freeman, "Ion Diffusion Coefficients in Ion Exchange Membranes: Significance of Counterion Condensation," Macromolecules, vol. 51, pp. 5519-5529, aug 2018.

[41] M. Seitanidou, K. Tybrandt, M. Berggren, and D. T. Simon, "Overcoming transport limitations in miniaturized electrophoretic delivery devices," Lab Chip, vol. 19, no. 8, pp. 1427-1455, 2019.

[42] N. Abdullayeva and M. Sankir, "Influence of electrical and ionic conductivities of organic electronic ion pump on acetylcholine exchange performance," Materials (Basel)., vol. 10, may 2017.

[43] D. T. Simon, S. Kurup, K. C. Larsson, R. Hori, K. Tybrandt, M. Goiny, E. W. Jager, M. Berggren, B. Canlon, and A. Richter-Dahlfors, "Organic electronics for precise delivery of neurotransmitters to modulate mammalian sensory function," Nat. Mater., vol. 8, no. 9, pp. 742-746, 2009.

[44] D. T. Simon, K. C. Larsson, D. Nilsson, G. Burström, D. Galter, M. Berggren, and A. Richter-Dahlfors, "An organic electronic biomimetic neuron enables auto-regulated neuromodulation," Biosens. Bioelectron., vol. 71, pp. 359-364, sep 2015.

[45] J.-H. Han, K. B. Kim, H. C. Kim, and T. D. Chung, "Ionic circuits based on polyelectrolyte diodes on a microchip.," Angew. Chemie, vol. 48, pp. 3830-3833, jan 2009 .

[46] K. Tybrandt, "Exploring the potential of ionic bipolar diodes for chemical neural interfaces," Soft Matter, vol. 13, pp. 8171-8177, nov 2017.

[47] R. A. Lucas, C. Y. Lin, L. A. Baker, and Z. S. Siwy, "Ionic amplifying circuits inspired by electronics and biology," Nat. Commun., vol. 11, no. 1, pp. 1-9, 2020 .

[48] G. Merle, M. Wessling, and K. Nijmeijer, "Anion exchange membranes for alkaline fuel cells: A review," Journal of Membrane Science, vol. 377, pp. 1-35, jul 2011. 
[49] C. T. Matos, R. Fortunato, S. Velizarov, M. A. Reis, and J. G. Crespo, "Removal of mono-valent oxyanions from water in an ion exchange membrane bioreactor: Influence of membrane permselectivity," Water Research, vol. 42, pp. 1785-1795, mar 2008 .

[50] C. Ponce de León, A. Frías-Ferrer, J. González-García, D. A. Szánto, and F. C. Walsh, "Redox flow cells for energy conversion," Journal of Power Sources, vol. 160, pp. 716-732, sep 2006.

[51] H. Strathmann, "Electrodialysis, a mature technology with a multitude of new applications," Desalination, vol. 264, pp. 268-288, dec 2010.

[52] Y. Tanaka, Chapter 7 Donnan Dialysis, vol. 12. 2007.

[53] G. D. Guerra, "24 - Electroinitiated Polymerization A2 - Allen, Geoffrey," Comprehensive Polymer Science and Supplements, pp. 453-458, 1989.

[54] J. E. Baur, "Diffusion Coefficients," Handbook of Electrochemistry, pp. 829-848, jan 2007.

[55] S. Kariuki and H. D. Dewald, "Evaluation of diffusion coefficients of metallic ions in aqueous solutions," Electroanalysis, vol. 8, pp. 307-313, apr 1996.

[56] W. F. W. F. Smith, "Foundations of materials science and engineering," 2003.

[57] T. Sata, "Ion Exchange Membranes," Ion Exchange Membranes, apr 2004.

[58] F. G. Donnan, "The Theory of Membrane Equilibria.," Chemical Reviews, vol. 1, pp. 73-90, apr 2002.

[59] A. Jonsson, ORGANIC ELECTRONICS FOR PRECISE DELIVERY OF NEUROTRANSMITTERS. PhD thesis, 2017.

[60] R.-w. Li and G. Liu, Flexible and Stretchable Electronics. CRC Press, 2018.

[61] . Klaus-Dieter Kreuer, *, §. Stephen J. Paddison, , . Eckhard Spohr, and M. Schuster $\ddagger$, "Transport in Proton Conductors for Fuel-Cell Applications: Simulations, Elementary Reactions, and Phenomenology," Chemical Reviews, vol. 104, pp. 4637-4678, oct 2004.

[62] T. Luo, S. Abdu, and M. Wessling, "Selectivity of ion exchange membranes: A review," J. Memb. Sci., vol. 555, pp. 429-454, jun 2018.

[63] N. Gnusin, N. Berezina, N. K. J. of membrane ..., and undefined 2004, "Transport structural parameters to characterize ion exchange membranes," Elsevier.

[64] V. I. Zabolotsky and V. V. Nikonenko, "Effect of structural membrane inhomogeneity on transport properties," Journal of Membrane Science, vol. 79, pp. 181198, may 1993. 
[65] N. Agmon, "The Grotthuss mechanism," Chemical Physics Letters, vol. 244, pp. 456-462, 1995.

[66] S. C. Ramírez and R. R. Paz, "Hydroxide Transport in Anion-Exchange Membranes for Alkaline Fuel Cells," New Trends in Ion Exchange Studies, nov 2018.

[67] H. Strathmann, A. Grabowski, and G. Eigenberger, "Ion-Exchange Membranes in the Chemical Process Industry," 2013.

[68] G. S. Gohil, V. V. Binsu, and V. K. Shahi, "Preparation and characterization of mono-valent ion selective polypyrrole composite ion-exchange membranes," Journal of Membrane Science, vol. 280, pp. 210-218, sep 2006.

[69] R. Femmer, A. Mani, and M. Wessling, "Ion transport through electrolyte/polyelectrolyte multi-layers," Scientific Reports 2015 5:1, vol. 5, pp. 1-12, jun 2015.

[70] C. Larson, B. Peele, S. Li, S. Robinson, M. Totaro, L. Beccai, B. Mazzolai, and R. Shepherd, "Highly stretchable electroluminescent skin for optical signaling and tactile sensing," tech. rep.

[71] J. H. Koo, D. C. Kim, J. Shim, T.-H. Kim, and D.-H. Kim, "Flexible and Stretchable Smart Display: Materials, Fabrication, Device Design, and System Integration," 2018.

[72] M. Hussain and N. El-Atab, Handbook of Flexible and Stretchable Electronics. CRC Press, 1 ed., 2019.

[73] T. Someya, Z. Bao, and G. G. Malliaras, "The rise of plastic bioelectronics," Nature, vol. 540, no. 7633, pp. 379-385, 2016.

[74] S. E. Root, S. Savagatrup, A. D. Printz, D. Rodriquez, and D. J. Lipomi, "Mechanical Properties of Organic Semiconductors for Stretchable, Highly Flexible, and Mechanically Robust Electronics," Chem. Rev., vol. 117, no. 9, pp. 64676499, 2017.

[75] N. Kim, S. Lienemann, I. Petsagkourakis, D. A. Mengistie, S. Kee, T. Ederth, V. Gueskine, P. Leclère, R. Lazzaroni, X. Crispin, and K. Tybrandt, "Elastic conducting polymer composites in thermoelectric modules," Nat. Commun. 2020 111, vol. 11, pp. 1-10, mar 2020.

[76] A. G. Evans, G. B. Crumley, and R. E. Demaray, "On the mechanical behavior of brittle coatings and layers," Oxid. Met., vol. 20, no. 5, pp. 193-216, 1983.

[77] R. J. Jaccodine and W. A. Schlegel, "Measurement of Strains at Si-SiO2 Interface," Journal of Applied Physics, vol. 37, no. 6, pp. 2429-2434, 1966.

[78] "JOHN WILEY \& SONS, Inc.," Ind. Eng. Chem., vol. 51, no. 6, pp. 89A-89A, 1959. 
[79] H. Gleskova, I.-C. Cheng, S. Wagner, J. C. Sturm, and Z. Suo, "Mechanics of thin-film transistors and solar cells on flexible substrates," Solar Energy, vol. 80, no. 6 , pp. 687-693, 2006.

[80] G. Janssen, M. M. Abdalla, F. van Keulen, B. R. Pujada, and B. van Venrooy, "Celebrating the 100th anniversary of the Stoney equation for film stress: Developments from polycrystalline steel strips to single crystal silicon wafers," Thin Solid Films, vol. 517, no. 6, pp. 1858-1867, 2009.

[81] S. P. Lacour, J. Jones, Z. Suo, and S. Wagner, "Design and Performance of Thin Metal Film Interconnects for Skin-Like Electronic Circuits," IEEE Electron Device Lett., vol. 25, no. 4, pp. 179-181, 2004.

[82] Z. Chen, B. Cotterell, and W. Wang, "The fracture of brittle thin films on compliant substrates in flexible displays," Eng. Fract. Mech., vol. 69, no. 5, pp. 597-603, 2002.

[83] C.-W. Kang and H. Huang, "Deformation, failure and removal mechanisms of thin film structures in abrasive machining," Adv. Manuf., vol. 5, no. 1, pp. 1-19, 2017.

[84] K. D. Harris, A. L. Elias, and H. J. Chung, "Flexible electronics under strain: a review of mechanical characterization and durability enhancement strategies," mar 2016.

[85] J. Song, "Mechanics of stretchable electronics," Current Opinion in Solid State and Materials Science, vol. 19, no. 3, pp. 160-170, 2015.

[86] A. Das, A. Banerji, and R. Mukherjee, "Programming Feature Size in the Thermal Wrinkling of Metal Polymer Bilayer by Modulating Substrate Viscoelasticity," ACS Appl. Mater. Interfaces, vol. 9, no. 27, pp. 23255-23262, 2017.

[87] T. Akter and W. S. Kim, "Reversibly Stretchable Transparent Conductive Coatings of Spray-Deposited Silver Nanowires," ACS Applied Materials Interfaces, vol. 4, no. 4, pp. 1855-1859, 2012.

[88] H. Lee, K. Lee, J. T. Park, W. C. Kim, and H. Lee, "Well-Ordered and High Density Coordination-Type Bonding to Strengthen Contact of Silver Nanowires on Highly Stretchable Polydimethylsiloxane," Advanced Functional Materials, vol. 24, no. 21, pp. 3276-3283, 2014.

[89] N. Karim, S. Afroj, S. Tan, P. He, A. Fernando, C. Carr, and K. S. Novoselov, "Scalable Production of Graphene-Based Wearable E-Textiles," ACS Nano, vol. 11, no. 12, pp. 12266-12275, 2017.

[90] T. Wang, R. Wang, Y. Cheng, and J. Sun, "Quasi In Situ Polymerization To Fabricate Copper Nanowire-Based Stretchable Conductor and Its Applications," ACS Appl. Mater. Interfaces, vol. 8, no. 14, pp. 9297-9304, 2016. 
[91] S. Choi, J. Park, W. Hyun, J. Kim, J. Kim, Y. B. Lee, C. Song, H. J. Hwang, J. H. Kim, T. Hyeon, and D.-H. Kim, "Stretchable Heater Using LigandExchanged Silver Nanowire Nanocomposite for Wearable Articular Thermotherapy," ACS Nano, vol. 9, no. 6, pp. 6626-6633, 2015.

[92] S.-H. Lee and Y.-J. Cho, "Characterization of Silver Inkjet Overlap-printing through Cohesion and Adhesion," J. Electr. Eng. Technol., vol. 7, no. 1, pp. 9196, 2012.

[93] F. Xu and Y. Zhu, "Highly Conductive and Stretchable Silver Nanowire Conductors," Advanced Materials, vol. 24, no. 37, pp. 5117-5122, 2012.

[94] J. Liang, L. Li, K. Tong, Z. Ren, W. Hu, X. Niu, Y. Chen, and Q. Pei, "Silver Nanowire Percolation Network Soldered with Graphene Oxide at Room Temperature and Its Application for Fully Stretchable Polymer Light-Emitting Diodes," ACS Nano, vol. 8, no. 2, pp. 1590-1600, 2014.

[95] J. Han, J. Lee, J. Lee, and J. Yeo, "Highly Stretchable and Reliable, Transparent and Conductive Entangled Graphene Mesh Networks," Adv. Mater., vol. 30, no. 3, p. $1704626,2017$.

[96] U.-H. Shin, D.-W. Jeong, S.-H. Kim, H. W. Lee, and J.-M. Kim, "ElastomerInfiltrated Vertically Aligned Carbon Nanotube Film-Based Wavy-Configured Stretchable Conductors," ACS Appl. Mater. Interfaces, vol. 6, no. 15, pp. 12909 12914, 2014.

[97] R. M. A. P. Lima, J. J. Alcaraz-Espinoza, F. A. G. da Silva, and H. P. de Oliveira, "Multifunctional Wearable Electronic Textiles Using Cotton Fibers with Polypyrrole and Carbon Nanotubes," ACS Applied Materials Interfaces, vol. 10, no. 16, pp. 13783-13795, 2018.

[98] Y.-Y. Lee, H.-Y. Kang, S. H. Gwon, G. M. Choi, S.-M. Lim, J.-Y. Sun, and Y.-C. Joo, "A Strain-Insensitive Stretchable Electronic Conductor: PEDOT:PSS/Acrylamide Organogels," Advanced Materials, vol. 28, no. 8, pp. 1636-1643, 2015.

[99] E. Song, B. Kang, H. H. Choi, D. H. Sin, H. Lee, W. H. Lee, and K. Cho, "Stretchable electronics: Stretchable and Transparent Organic Semiconducting Thin Film with Conjugated Polymer Nanowires Embedded in an Elastomeric Matrix (Adv. Electron. Mater. 1/2016)," Advanced Electronic Materials, vol. 2, no. $1,2016$.

[100] F. Hu, Y. Xue, J. Xu, and B. Lu, "PEDOT-Based Conducting Polymer Actuators," Frontiers in Robotics and AI, vol. 6, p. 114, nov 2019.

[101] Y. Zhou, C. Wan, Y. Yang, H. Yang, S. Wang, Z. Dai, K. Ji, H. Jiang, X. Chen, and Y. Long, "Highly Stretchable, Elastic, and Ionic Conductive Hydrogel for Artificial Soft Electronics," Adv. Funct. Mater., vol. 29, no. 1, p. 1806220, 2018. 
[102] J.-Y. Sun, X. Zhao, W. R. K. Illeperuma, O. Chaudhuri, K. H. Oh, D. J. Mooney, J. J. Vlassak, and Z. Suo, "Highly stretchable and tough hydrogels," Nature 2012 489:7414, vol. 489, pp. 133-136, sep 2012.

[103] J. Kamcev, D. R. Paul, and B. D. Freeman, "Effect of fixed charge group concentration on equilibrium ion sorption in ion exchange membranes," Journal of Materials Chemistry A, vol. 5, no. 9, pp. 4638-4650, 2017.

[104] f. given $i=J$. S., given=Jae Sung, f. given $i=M$., given=Mohamad, and f. given $\mathrm{i}=\mathrm{W}$. S., given=Woo Soo, "Sustained percolation in stretched silver nanowire networks for stretchable inter-connection applications," vol. 16, no. 7, pp. 905908.

[105] f. given $\mathrm{i}=\mathrm{G}$. D., given=Geon Dae, f. given $\mathrm{i}=\mathrm{G}$. H., given=Guh-Hwan, f. given $\mathrm{i}=\mathrm{J}$. H., given=Jun Hyuk, f. given $\mathrm{i}=\mathrm{M}$., given=Minkwan, f. given $\mathrm{i}=\mathrm{T}$., given=Taekyung, f. given $\mathrm{i}=\mathrm{B}$., given=Byungkwon, and f. given $\mathrm{i}=\mathrm{U}$., given=Unyong, "Highly stretchable patterned gold electrodes made of au nanosheets," vol. 25, no. 19, pp. 2707-2712.

[106] B. Cotterell and Z. Chen, "Buckling and Fracture of Thin Films under Compression," Key Eng. Mater., vol. 183, pp. 187-192, 2000.

[107] Z. Jiang, K. Fukuda, X. Xu, S. Park, D. Inoue, H. Jin, M. Saito, I. Osaka, K. Takimiya, and T. Someya, "Transparent Electrodes: Reverse-Offset Printed Ultrathin Ag Mesh for Robust Conformal Transparent Electrodes for High-Performance Organic Photovoltaics (Adv. Mater. 26/2018)," Adv. Mater., vol. 30, no. 26, p. 1870190, 2018.

[108] K. Sim, Y. Li, J. Song, and C. Yu, "Stretchable Electronics: Biaxially Stretchable Ultrathin Si Enabled by Serpentine Structures on Prestrained Elastomers (Adv. Mater. Technol. 1/2019)," Advanced Materials Technologies, vol. 4, no. 1, p. 1970003, 2019.

[109] Y. Won, A. Kim, W. Yang, S. Jeong, and J. Moon, "A highly stretchable, helical copper nanowire conductor exhibiting a stretchability of $700 \%$," $N P G$ Asia Mater., vol. 6, no. 9, pp. e132-e132, 2014.

[110] M. W. Woo, P. Wong, Y. Tang, V. Triacca, P. E. Gloor, A. N. Hrymak, and A. E. Hamielec, "Melting behavior and thermal properties of high density polythylene," Polymer Engineering Science, vol. 35, pp. 151-156, jan 1995.

[111] T. H. Rieckmann and S. V. . Olker, "2 Poly(Ethylene Terephthalate) Polymerization-Mechanism, Catalysis, Kinetics, Mass Transfer and Reactor Design,"

[112] M. A. Riheen, T. K. Saha, and P. K. Sekhar, "Inkjet Printing on PET Substrate," Journal of The Electrochemical Society, vol. 166, p. B3036, mar 2019. 
[113] C. Qu, J. Hu, X. Liu, Z. Li, and Y. Ding, "Morphology and Mechanical Properties of Polyimide Films: The Effects of UV Irradiation on Microscale Surface," Materials, vol. 10, no. 11, p. 1329, 2017.

[114] H. Deligöz, T. Yalcinyuva, S. Özgümüs, and S. Yildirim, "Electrical properties of conventional polyimide films: Effects of chemical structure and water uptake," Journal of Applied Polymer Science, vol. 100, no. 1, pp. 810-818, 2006.

[115] J. C. Lötters, W. Olthuis, P. H. Veltink, and P. Bergveld, "The mechanical properties of the rubber elastic polymer polydimethylsiloxane for sensor applications," J. Micromechanics Microengineering, vol. 7, pp. 145-147, sep 1997.

[116] I. D. Johnston, D. K. McCluskey, C. K. L. Tan, and M. C. Tracey, "Mechanical characterization of bulk Sylgard 184 for microfluidics and microengineering," $J$. Micromechanics Microengineering, vol. 24, p. 035017, feb 2014.

[117] A. Victor, J. Ribeiro, and F. F. Araújo, "Study of PDMS characterization and its applications in biomedicine: A review," J. Mech. Eng. Biomech., vol. 4, pp. 1-9, aug 2019.

[118] H. Kim, M. Chandran, S. Park, H. Chae, J. Lee, and Y. Choe, "Electrical properties of polymer photovoltaic cells using pentacene-doped PEDOT: PSS as a hole conducting layer," Surface and Interface Analysis, vol. 44, no. 11-12, pp. 1511-1514, 2012.

[119] W. Quirós-Solano, N. Gaio, C. Silvestri, G. Pandraud, and P. Sarro, "PEDOT:PSS: A Conductive and Flexible Polymer for Sensor Integration in Organon-Chip Platforms," Procedia Engineering, vol. 168, pp. 1184-1187, 2016.

[120] T. Abrahamsson, D. J. Poxson, E. O. Gabrielsson, M. Sandberg, D. T. Simon, and M. Berggren, "Formation of Monolithic Ion-Selective Transport Media Based on "Click" Cross-Linked Hyperbranched Polyglycerol," Frontiers in Chemistry, vol. 7, p. 484, jul 2019.

[121] D. J. Poxson, E. O. Gabrielsson, A. Bonisoli, U. Linderhed, T. Abrahamsson, I. Matthiesen, K. Tybrandt, M. Berggren, and D. T. Simon, "Capillary-Fiber Based Electrophoretic Delivery Device," ACS Appl. Mater. Interfaces, vol. 11, pp. 14200-14207, apr 2019.

[122] T. Arbring Sjöström, A. Jonsson, E. O. Gabrielsson, L. Kergoat, K. Tybrandt, M. Berggren, and D. T. Simon, "Cross-Linked Polyelectrolyte for Improved Selectivity and Processability of Iontronic Systems," ACS Appl. Mater. Interfaces, vol. 9, pp. 30247-30252, sep 2017.

[123] J. Suikkola, T. Björninen, M. Mosallaei, T. Kankkunen, P. Iso-Ketola, L. Ukkonen, J. Vanhala, and M. Mäntysalo, "Screen-Printing Fabrication and Characterization of Stretchable Electronics," Scientific Reports, vol. 6, no. 1, 2016. 
[124] P. He, J. Cao, H. Ding, C. Liu, J. Neilson, Z. Li, I. A. Kinloch, and B. Derby, "Screen-Printing of a Highly Conductive Graphene Ink for Flexible Printed Electronics," ACS Applied Materials Interfaces, vol. 11, no. 35, pp. 32225$32234,2019$.

[125] G. Dubey, "Screens for screen printing of electronic circuits," Microelectronics Reliability, vol. 13, no. 3, pp. 203-207, 1974.

[126] A. Willfahrt, Screen Printing Technology for Energy Devices. No. 1942, 2019.

[127] H. Sirringhaus and T. Shimoda, "Inkjet Printing of Functional Materials," MRS Bull., vol. 28, no. 11, pp. 802-806, 2003.

[128] M. Singh, H. M. Haverinen, P. Dhagat, and G. E. Jabbour, "Inkjet PrintingProcess and Its Applications," Adv. Mater., vol. 22, no. 6, pp. 673-685, 2010.

[129] I. M. Hutchings and G. D. Martin, Inkjet Technology for Digital Fabrication. c 2013 John Wiley \& Sons Ltd, 2013.

[130] G. Faraji, H. S. Kim, and H. T. Kashi, "Introduction," in Severe Plastic Deformation, pp. 1-17, Elsevier, jan 2018.

[131] A. Lasia, "Electrochemical Impedance Spectroscopy and its Applications," Modern Aspects of Electrochemistry, pp. 143-248, nov 2002.

[132] J. W. Lichtman and J. A. Conchello, "Fluorescence microscopy," nov 2005. 
Journal articles 


\section{Papers}

The papers associated with this thesis have been removed for copyright reasons. For more details about these see:

http://urn.kb.se/resolve?urn=urn:nbn:se:liu:diva-179010 


\section{FACULTY OF SCIENCE AND ENGINEERING}

Linköping Studies in Science and Technology, Dissertation No. 2174, 2021

Department of Science and Technology

Linköping University

SE-581 83 Linköping, Sweden

www.liu.se 